\title{
On algebraic construction of certain integrable and super-integrable systems
}

\author{
A. J. Maciejewski ${ }^{1}$, M. Przybylska ${ }^{2}$, A. V. Tsiganov ${ }^{3}$ \\ ${ }^{1} \mathrm{~J}$. Kepler Institute of Astronomy, University of Zielona Góra, Licealna 9, \\ PL-65-417 Zielona Góra, Poland (e-mail: maciejka@astro.ia.uz.zgora.pl) \\ ${ }^{2}$ Institute of Physics, University of Zielona Góra, Licealna 9, \\ PL-65-417 Zielona Góra, Poland \\ (e-mail:M.Przybylska@proton.if.uz.pl) \\ ${ }^{3}$ St. Petersburg State University, St. Petersburg, Russia \\ (e-mail: andrey.tsiganov@gmail.com)
}

\begin{abstract}
We propose a new construction of two-dimensional natural bi-Hamiltonian systems associated with a very simple Lie algebra. The presented construction allows us to distinguish three families of super-integrable monomial potentials for which one additional first integral is quadratic, and the second one can be of arbitrarily high degree with respect to the momenta. Many integrable systems with additional integrals of degree greater than two in momenta are given. Moreover, an example of a superintegrable system with first integrals of degree two, four and six in the momenta is found.
\end{abstract}

\section{Introduction}

The main aim of this paper is to study natural integrable systems

$$
H_{1}=2 p_{1} p_{2}+V\left(q_{1}, q_{2}\right)
$$

with two degrees of freedom associated with the various representations of Lie algebra

$$
\left[N, a_{+}\right]=\kappa_{1} a_{+}, \quad\left[N, a_{-}\right]=-\kappa_{2} a_{-}, \quad\left[a_{+}, a_{-}\right]=0
$$

labelled by parameters $\kappa_{1}, \kappa_{2} \in \mathbb{R}$, and having the Casimir element

$$
C=a_{+}^{k_{2}} a_{-}^{\kappa_{1}} .
$$

An obvious representation of this algebra is the algebra of smooth function defined on the phase space generated by

$$
a_{+}=q_{1}, \quad a_{-}=q_{2}, \quad N=-\kappa_{1} q_{1} p_{1}+\kappa_{2} q_{2} p_{2},
$$

with bracket $[a, b]:=\{a, b\}$, where $\{\cdot, \cdot\}$ denotes the canonical Poisson bracket in $\mathbb{R}^{4}$. 
We met with this algebra investigating bi-Hamiltonian structures of two-dimensional natural Hamiltonian systems with homogeneous potentials and Newton's equations with homogeneous velocity independent forces. Namely, let us consider a natural system given by the Hamilton function of the form (1.1) with a monomial potential. More precisely, let us consider system governed by Hamiltonian of the form

$$
H \equiv a_{+}=2 p_{1} p_{2}+q_{1}^{a} q_{2}^{b},
$$

where $a$ and $b$ are real parameters. For such a system the function

$$
N=\alpha p_{1} q_{1}+\beta p_{2} q_{2},
$$

satisfies the following equality

$$
\left\{N, a_{+}\right\}=(\alpha+\beta) a_{+},
$$

provided that $\alpha$ and $\beta$ are fulfils equation

$$
a \alpha+b \beta+\alpha+\beta=0 .
$$

If we can find the remaining generator $a_{-}=H_{2}$, then we have integrable in the Liouville sense dynamical system with homogeneous potential. In order to get this additional first integral we use the machinery of bi-Hamiltonian geometry, see [22, 26].

It is worth to notice that the existence of function $N$ with the prescribed property is related with a certain symmetry of the system. Let $X_{F}$ denote the Hamiltonian vector field generated by $F$. Assume that for Hamiltonian (1.4) there exists function $N$ such that $\{N, H\}=c H$, where $c$ is a constant. Then the Hamiltonian vector field $X_{N}$ is a master symmetry of $X_{H}$, as $\left[X_{N}, X_{H}\right]=c X_{H}$. Sometimes a master symmetry is called a conformal symmetry, see [3].

Let we assume that $X_{N}$ with $N$ of the following form

$$
N:=A\left(q_{1}, q_{2}\right) p_{1}+B\left(q_{1}, q_{2}\right) p_{2},
$$

where $A\left(q_{1}, q_{2}\right)$ and $B\left(q_{1}, q_{2}\right)$ are differentiable functions, is a conformal symmetry of $X_{H}$. Then it is easy to show that necessarily $A=\alpha q_{1}$ and $B=\beta q_{2}$. Thus, equality $\{N, H\}=c H$ implies that

$$
c=\alpha+\beta, \quad \alpha a+\beta b+c=0 .
$$

So, we recovered the assumed form of $N$, see (1.5). Now, the last relation in (1.2) tells that we need an additional integral $H_{2} \equiv a_{-}$. Moreover, we require that $\left\{N, H_{2}\right\}=d H_{2}$, for a certain $d \in \mathbb{R}$, and this is equivalent that $X_{N}$ is also a conformal symmetry of $X_{H_{2}}$.

We are going to work with systems (1.4) with two degrees of freedom because such systems appeared as subsystems on invariant manifold of $n$-dimensional Hamiltonian systems [13, 19].

The plan of this paper is following. In the next section we start with short description how Hamiltonian systems of the form (1.4) appeared in our investigations of the integrability of natural Hamiltonian systems with homogeneous potentials. In Section 3 bi-Hamiltonian irregular Poisson manifolds as well as their application for construction of first integrals of considered systems are presented. The remaining sections contain results 
of the integrability analysis. In Section 4 four families of integrable systems with additional first integrals quadratic in the momenta are given. In the next four sections super-integrable cases in these families are distinguished. In Section 9 examples of integrable systems with additional first integrals of degree greater than two in the momenta are given. In Appendix we collected basic facts concerning the Gauss hypergeometric equation which are used in this paper.

\section{Monomial potentials}

Let us consider Hamiltonian systems with $n$ degrees of freedom with the Hamiltonian of the following classical form

$$
H=\frac{1}{2} \sum_{i=1}^{n} p_{i}^{2}+V(\boldsymbol{q})
$$

where $\boldsymbol{q}=\left(q_{1}, \ldots, q_{n}\right)$ and $\boldsymbol{p}=\left(p_{1}, \ldots, p_{n}\right)$ are the canonical coordinates and momenta; the potential $V(\boldsymbol{q})$ is a homogeneous function of degree $k \in \mathbb{Q}$. The strongest necessary integrability conditions for such systems with $k \in \mathbb{Z}$ were obtained thanks to an application of differential Galois methods, see [15, 16, 11]. To derive conditions of this type one needs to know a particular non-equilibrium solution of the considered systems. For the systems given by Hamiltonian (2.1) a particular solution can be find in systematic way. Namely, if a non-zero $d \in \mathbb{C}^{n}$ satisfies

$$
V^{\prime}(d)=\gamma d,
$$

for some $\gamma \in \mathbb{C}$, then the system has a particular solution of the following form

$$
\boldsymbol{q}(t)=\varphi(t) \boldsymbol{d}, \quad \boldsymbol{p}(t)=\dot{\varphi}(t) \boldsymbol{d},
$$

where $\varphi(t)$ is a scalar function which is defined in the following way. If vector $d$ satisfies equation (2.2) with $\gamma=0$, then $\varphi(t):=t$, and $d$ is called an improper Darboux point of potential $V$. In this case, if $k \in \mathbb{N}$, and the system is integrable in the Liouville sense, then all the eigenvalues of the Hessian matrix $V(\boldsymbol{d})$ vanish, see [19].

If the considered $\boldsymbol{d}$ satisfies equation (2.2) with $\gamma \neq 0$, then $\varphi(t)$ is a solution of equation $\ddot{\varphi}=-\varphi^{k-1}$. In this case $d$ is called a proper Darboux point of $V$, and if the system is integrable, then for a given $k \in \mathbb{Z} \backslash\{-2,2\}$, all eigenvalues of $V^{\prime \prime}(\boldsymbol{d})$ belong to a certain infinite subset of rational numbers $\mathbb{Q}$, see [15, 16, 13].

As we can see, in spite of the fact that the differential Galois theory is quite involved the final result has the form of simple arithmetic restrictions on the eigenvalues of matrix $V^{\prime \prime}(\boldsymbol{d})$. Moreover, for polynomial potentials some relations between eigenvalues of the Hessian calculated at all proper Darboux points exist. These relations together with arithmetic restrictions on the eigenvalues forced by necessary integrability conditions enable to find effectively explicit forms of integrable potentials at least for small $n$ and $k$. Such a systematic analysis was initiated for $n=2$ in [10, 11] and later on it was developed for $n>2$, see [19, 20].

However, for some classes of potentials the above approach does not work. In particular it is a case if the consider potential does not have any proper Darboux point, and moreover, the Hessian matrix $V^{\prime \prime}(\boldsymbol{d})$ at each improper Darboux point is nilpotent. For $n=2$ an almost complete characterisation of such polynomial potentials is given by the following lemma. 
Lemma 2.1. If a polynomial potential $V$ of degree $k>2$ does not have any proper Darboux point, then it is either equivalent to the following one

$$
V_{k, l}=\alpha\left(q_{2}-\mathrm{i} q_{1}\right)^{k-l}\left(q_{2}+\mathrm{i} q_{1}\right)^{l}, \quad \alpha \in \mathbb{C}^{\star}:=\mathbb{C} \backslash\{0\} .
$$

for some $l=2, \ldots, k-2$, or $k=2 s$ and $V$ has factor $\left(q_{2} \pm \mathrm{i} q_{1}\right)$ with multiplicity $s$.

We say that potential $V$ is equivalent to $W$ iff $V(\boldsymbol{q})=W(A \boldsymbol{q})$, where $A$ is $n \times n$ matrix satisfying $A A^{T}=\beta \operatorname{Id}_{n}$ for a certain $\beta \in \mathbb{C}^{\star}$.

Let us notice that potential $V_{k, l}$ with $2 \leq l \leq k-2$ has two improper Darboux points $\boldsymbol{d}_{1}=(1, \mathrm{i})$ and $\boldsymbol{d}_{2}=(1,-\mathrm{i})$. Moreover, $V^{\prime \prime}\left(\boldsymbol{d}_{1}\right)$ and $V^{\prime \prime}\left(\boldsymbol{d}_{2}\right)$ are nilpotent. In other words, for these potentials we do not have any obstacles for the integrability.

The potentials of the form (2.4) look more attractive if we introduce new canonical variables

$$
z_{1}=q_{1}+\mathrm{i} q_{2}, \quad z_{2}=q_{1}-\mathrm{i} q_{2}, \quad y_{1}=\frac{1}{2}\left(p_{1}-\mathrm{i} p_{2}\right), \quad y_{2}=\frac{1}{2}\left(p_{1}+\mathrm{i} p_{2}\right) .
$$

In these variables the Hamiltonian function has the form

$$
H=2 y_{1} y_{2}+z_{1}^{l} z_{2}^{k-l} .
$$

Clearly, the systems governed by Hamiltonian function of the form (1.4) are just generalisation of (2.6) for cases when $k$ and $l$ are rational.

\section{Irregular Poisson manifolds}

A bi-Hamiltonian manifold $M$ is a smooth manifold endowed with a pair of compatible Poisson bi-vectors $P$ and $P^{\prime}$ such that

$$
\llbracket P, P^{\prime} \rrbracket=0, \quad \llbracket P^{\prime}, P^{\prime} \rrbracket=0,
$$

where $\llbracket \cdot, \cdot \rrbracket$ is the Schouten bracket. If $\operatorname{dim} M=2 n$, and $P$ is invertible Poisson bivector on $M$, the Nijenhuis operator which is called also the hereditary, or recursion operator, is defined as

$$
\mathcal{N}=P^{\prime} P^{-1} \text {. }
$$

We say that a bi-Hamiltonian manifold $M$ is regular iff $\operatorname{dim} M=2 n, P$ is invertible Poisson bivector, and the recursion operator $\mathcal{N}$ has, at every point, $n$ distinct functionally independent eigenvalues. If the recursion operator $\mathcal{N}$ does not have this property then we say that bi-Hamiltonian manifold $M$ is irregular.

For a regular bi-Hamiltonian manifold $M$ functions

$$
H_{k}=\frac{1}{2 k} \operatorname{Tr} \mathcal{N}^{k}, \quad \text { for } \quad k \in \mathbb{N},
$$

form a bi-Hamiltonian hierarchy on $M$, i.e., the Lenard relations hold

$$
P^{\prime} \mathrm{d} H_{k}=P \mathrm{~d} H_{k+1}, \quad \text { for all } k \geq 1 \text {. }
$$

Generally it is unknown if it is possible to construct in a systematic way a full involutive set of functions on irregular bi-Hamiltonian manifolds.

Anyway, we are going to apply the algebra (1.2) to construct integrable systems on two-dimensional irregular bi-Hamiltonian manifolds. 


\subsection{The rational Calogero-Moser system}

In order to justify better some aspects of our construction we consider the $n$-particle rational Calogero-Moser model associated with the root system $\mathcal{A}_{n}$. It is defined by the Hamilton function

$$
H=\frac{1}{2} \sum_{i=1}^{n} p_{i}^{2}-a^{2} \sum_{i \neq j}^{n} \frac{1}{\left(q_{i}-q_{j}\right)^{2}}
$$

where $a$ is a coupling constant. The canonical variables $(\boldsymbol{q}, \boldsymbol{p})$ satisfy the standard Poisson bracket relations $\left\{q_{i}, p_{j}\right\}=\delta_{i j}$, and the associated Poisson bivector will be denoted by $P$.

This system admits independent and commuting first integrals

$$
H_{k}=\frac{1}{k !} \operatorname{Tr} L^{k}, \quad \text { for } k=1, \ldots, n,
$$

where $L$ is the standard Lax matrix

$$
L=\left[\begin{array}{cccc}
p_{1} & \frac{a}{q_{1}-q_{2}} & \cdots & \frac{a}{q_{1}-q_{n}} \\
\frac{a}{q_{2}-q_{1}} & p_{2} & \ddots & \vdots \\
\vdots & \ddots & \ddots & \vdots \\
\frac{a}{q_{n}-q_{1}} & \frac{a}{q_{n}-q_{2}} & \cdots & p_{n}
\end{array}\right] .
$$

Besides these $n$ integrals of motion the rational Calogero-Moser system admits $(n-1)$ additional functionally independent integrals of motion $K_{m}$ given by

$$
K_{m}=m g_{1} H_{m}-g_{m} H_{1}, \quad m=2, \ldots, n,
$$

where

$$
g_{j}=\frac{1}{2}\left\{\sum_{i=1}^{n} q_{i}^{2}, H_{j}\right\}, \text { for } j=1, \ldots, n .
$$

According to [26] integrals of motion $H_{k}$ and $K_{m}$ can be obtained from the Hamilton function $\mathrm{H}=\mathrm{H}_{2}$ given by (3.4) as polynomial in momenta solutions of the following equations

$$
P \mathrm{~d} H=k^{-1} P^{\prime} \mathrm{d} \ln H_{k}=(m-1)^{-1} P^{\prime} \mathrm{d} \ln K_{m},
$$

where $P^{\prime}$ is the Poisson bivector compatible with $P$ given by

$$
P^{\prime}=\left[\begin{array}{cc}
R & \Pi \\
-\Pi & 0
\end{array}\right]+\left[\begin{array}{cc}
0 & \Lambda \\
-\Lambda & M
\end{array}\right]
$$

and $n \times n$ matrices $\Pi, \Lambda, R$ and $M$ have the following entries

$$
\Pi_{i j}=p_{i} p_{j}, \quad \Lambda_{i j}=q_{i} \sum_{k \neq j}^{n} \frac{a^{2}}{\left(q_{j}-q_{k}\right)^{3}},
$$

and

$$
R_{i j}=\sum_{k=1}^{n}\left(\frac{\partial \Pi_{j k}}{\partial p_{i}}-\frac{\partial \Pi_{i k}}{\partial p_{j}}\right) q_{k}, \quad M_{i j}=\sum_{k=1}^{n}\left(\frac{\partial \Lambda_{k i}}{\partial q_{j}}-\frac{\partial \Lambda_{k j}}{\partial q_{i}}\right) p_{k} .
$$


In this case recursion operator $\mathcal{N}=P^{\prime} P^{-1}$ generates only the Hamilton function

$$
\operatorname{Tr} \mathcal{N}^{k}=2(2 H)^{k} \quad \text { such that } \quad P \mathrm{~d} H=P^{\prime} \mathrm{d} \ln H,
$$

and instead of the standard Lenard relations (3.3) we have equations (3.5). Thus, the described Calogero-Moser system is a super-integrable system on an irregular bi-Hamiltonian manifold $\mathbb{R}^{2 n}$.

\subsection{Algebraic construction of two-dimensional bi-Hamiltonian systems on ir- regular manifolds}

In order to get integrable systems associated with the Lie algebra (1.2) we start with the canonical Poisson bivector

$$
P=\left[\begin{array}{cccc}
0 & 0 & 1 & 0 \\
0 & 0 & 0 & 1 \\
-1 & 0 & 0 & 0 \\
0 & -1 & 0 & 0
\end{array}\right]
$$

and the Hamiltonian vector field

$$
X=P d H_{1}=\left[2 p_{2}, 2 p_{1},-\frac{\partial V\left(q_{1}, q_{2}\right)}{\partial q_{1}},-\frac{\partial V\left(q_{1}, q_{2}\right)}{\partial q_{2}}\right]^{T} .
$$

We suppose that this vector field $X$ is the bi-Hamiltonian vector field with respect to a certain Poisson bivector $P^{\prime}$, and some second integral of motion $\mathrm{H}_{2}$

$$
X=P d H_{1}=P^{\prime} \mathrm{d} H_{2} .
$$

The bivector $P^{\prime}$ has to be compatible with canonical bivector $P$, and it has to be the Poisson bivector. Hence, we have the following relations

$$
\llbracket P, P^{\prime} \rrbracket=0, \quad \llbracket P^{\prime}, P^{\prime} \rrbracket=0, \quad \text { and } \quad\left\{H_{1}, H_{2}\right\}=\left\{H_{1}, H_{2}\right\}^{\prime}=0 .
$$

Additionally we postulate that bivector $P^{\prime}$ is a Lie derivative of canonical bivector $P$ along the vector field $Y$ proportional to the Hamiltonian vector field $X$

$$
P^{\prime}=\mathcal{L}_{Y} P, \quad Y=\rho X,
$$

where multiplier $\rho$ is equal to

$$
\rho=\alpha p_{1} q_{1}+\beta p_{2} q_{2}
$$

and so it coincides with $N$ in (1.5).

Assumption (3.8) ensures that $P$ and $P^{\prime}$ are compatible bivectors, i.e., $\llbracket P, P^{\prime} \rrbracket=0$, and that eigenvalues of the corresponding recursion operator $\mathcal{N}=P^{\prime} P^{-1}$ are functions which depend only on $H_{1}$. In fact, we have

$$
\operatorname{det}\left(\mathcal{N}-\lambda \operatorname{Id}_{4}\right)=\lambda^{2}\left(\lambda+(\alpha+\beta) H_{1}\right)^{2},
$$

see (3.6). Thus, we have constructed an irregular bi-Hamiltonian manifold $\mathbb{R}^{4}$. 
Theorem 3.1. Bivector $P^{\prime}(\underline{3.8)}$ satisfies the Jacobi condition

$$
\llbracket P^{\prime}, P^{\prime} \rrbracket=0
$$

iff

$$
\alpha=0, \quad V=\frac{f\left(q_{1}\right)}{q_{2}}, \quad \rho=\beta p_{2} q_{2}
$$

or

$$
V=\left(\gamma+g\left(q_{1}^{-\beta / \alpha} q_{2}\right)\right) q_{1}^{-(\alpha+\beta) / \alpha}, \quad \rho=\alpha p_{1} q_{1}+\beta p_{2} q_{2},
$$

where $f$ and $g$ are arbitrary smooth functions, $\alpha \neq 0, \beta$ and $\gamma$ are arbitrary numbers.

Proof. In order to prove this theorem we have to substitute the second bivector

$$
P^{\prime}=\mathcal{L}_{Y} P=\left[\begin{array}{cccc}
0 & -2 \alpha p_{1} q_{1}+2 \beta p_{2} q_{2} & -2 \alpha p_{1} p_{2}+\alpha q_{1} \frac{\partial V}{\partial q_{1}} & -2 \beta p_{2}^{2}+\alpha q_{1} \frac{\partial V}{\partial q_{2}} \\
* & 0 & -2 \alpha p_{1}^{2}+\beta q_{2} \frac{\partial V}{\partial q_{1}} & -2 \beta p_{1} p_{2}+\beta q_{2} \frac{\partial V}{\partial q_{2}} \\
* & * & 0 & -\alpha p_{1} \frac{\partial V}{\partial q_{2}}+\beta p_{2} \frac{\partial V}{\partial q_{1}} \\
* & * & * & 0
\end{array}\right]
$$

into the Schouten bracket (3.9), and to solve the resulting system of partial differential equations.

Theorem 3.2. Hamilton function $H_{1}$ defined by (1.1) with potential $V$ of the form (3.10) or (3.11) satisfies the following relations

$$
\left\{H_{1}, \rho\right\}=-(\alpha+\beta) H_{1} \text {, }
$$

and

$$
\left\{H_{1}, \rho\right\}^{\prime}=\left(-(\alpha+\beta) H_{1}\right)^{2}
$$

and

$$
X=P \mathrm{~d} H_{1}=-(\alpha+\beta)^{-1} P^{\prime} \mathrm{d} \ln H_{1} .
$$

This therem is a direct consequence of the special form of $P^{\prime}$ and $H_{1}$.

Inspired by the equations (3.5) for the Calogero-Moser super-integrable system we suppose that our systems are bi-Hamiltonian systems with respect to logarithm of the second integral of motion $\mathrm{H}_{2}$, i.e.,

$$
X=P d H_{1}=\kappa_{2}^{-1} P^{\prime} \mathrm{d} \ln H_{2} .
$$

This additional assumption is equivalent to the requirement that $H_{2}$ and $\rho$ satisfy supplementary relations

$$
\left\{H_{2}, \rho\right\}=\kappa_{2} H_{2}, \quad \text { and } \quad\left\{H_{2}, \rho\right\}^{\prime}=-(\alpha+\beta) \kappa_{2} H_{1} H_{2} .
$$

The obtained relations (3.13), (3.14) and (3.17) show that generators $H_{1}, H_{2}$ and $\rho$ form linear and quadratic Poisson algebras with respect to the brackets $\{\cdot, \cdot\}$ and $\{\cdot, \cdot\}^{\prime}$, respectively. 
REMARK 3.3 Assumptions (3.8) and (3.16) yield the representation of the algebra (1.2), but we can derive (3.16) from the algebraic relations (1.2) only if (3.8) holds. Of course, form of (3.16) is a little strange because the logarithm of an integral of motion is itself integral of motion.

The equation (3.16) is equivalent to the following system of the partial differential equations

$$
\kappa_{2} H_{2}\left[2 p_{2}, 2 p_{1},-\frac{\partial V}{\partial q_{1}},-\frac{\partial V}{\partial q_{2}}\right]^{T}=P^{\prime}\left[\frac{\partial H_{2}}{\partial q_{1}}, \frac{\partial H_{2}}{\partial q_{2}}, \frac{\partial H_{2}}{\partial p_{1}}, \frac{\partial H_{2}}{\partial p_{2}}\right]^{T},
$$

with respect to unknown function $H_{2}=H_{2}\left(p_{1}, p_{2}, q_{1}, q_{2}\right)$, parameters $\alpha, \beta, \kappa_{2}$ and functions $f\left(q_{1}\right)$ or $g\left(q_{1}^{-\beta / \alpha} q_{2}\right)$ which appear in definition of potential $V\left(q_{1}, q_{2}\right)$ given by $(\underline{3.10})$, or (3.11). The explicit form of $P^{\prime}$ appearing in (3.18) is given by (3.12).

REMARK 3.4 Sometimes equations (3.18) have different functionally independent solutions $\mathrm{H}_{2}$ associated with the same potential $V$. In this case we obtain the so-called super-integrable system [24. 25], and our algebra (1.2) is some subalgebra of the complete polynomial algebra of integrals of motion.

In order to get integral of motion $\mathrm{H}_{2}$ we additionally assume that it is polynomial in momenta, i.e., it has the form

$$
H_{2}=\sum_{k, m=0}^{M} g_{k m}\left(q_{1}, q_{2}\right) p_{1}^{m} p_{2}^{k}
$$

where $g_{k m}\left(q_{1}, q_{2}\right)$ are smooth functions. Substituting this expression into (3.18) we obtain partial differential equations for unknown functions $g_{k m}, f, g$, and parameters $\alpha, \beta$. We can solve them for a fixed value of $M$. For instance, in the cubic case $M=3$, for $\alpha=0$ and $\kappa_{2}=0$, we obtain the following solution

$$
\rho=-q_{2} p_{2}, \quad V=\frac{\left(c_{1} q_{1}+c_{2}\right)^{-1}}{q_{2}}, \quad H_{2}=H_{1}\left(\frac{c_{1} q_{1}+c_{2}}{c_{1}} p_{1}+\rho\right) .
$$

Of course, the Hamiltonian

$$
H_{1}=2 p_{1} p_{2}+\frac{\left(c_{1} q_{1}+c_{2}\right)^{-1}}{q_{2}}
$$

possesses the first integral

$$
I_{2}=\frac{c_{1} q_{1}+c_{2}}{c_{1}} p_{1}-q_{2} p_{2}
$$

which is linear in the momenta. However, this first integral $I_{2}$ does not satisfy (3.18) in contrast with cubic integral $\mathrm{H}_{2}$.

In the next step, if we look for a first integral of degree $M=4$ in the momenta, then for $\alpha=0$, we reproduce previous solution and get one new

$$
\rho=-p_{2} q_{2}, \quad V=\frac{f}{q_{2}}, \quad f=\left(c_{1} q_{1}^{2}+c_{2} q_{1}+c_{3}\right)^{-1 / 2},
$$

associated with the fourth order in momenta integral of motion

$$
H_{2}=-\frac{2 p_{1} \rho+f}{4}\left(c_{1} \rho^{2}+\frac{f p_{1}^{2}+\left(2 p_{1} \rho+f\right) f^{\prime}}{q_{2} f^{3}}\right) .
$$


The obtained potential $V$ is non-polynomial and non-homogeneous function. Below we will select from generic solutions certain particular solutions which are given by homogeneous functions.

\section{Bi-Hamiltonian systems with second order integrals of motion}

Let us consider potential $V\left(q_{1}, q_{2}\right)$ given by (3.11) with

$$
\gamma=0, \quad \text { and } \quad g(z)=z^{d} .
$$

That is

$$
V\left(q_{1}, q_{2}\right)=q_{1}^{-\frac{\beta(d+1)+\alpha}{\alpha}} q_{2}^{d}
$$

and so, in this case the Hamiltonian has the form

$$
H_{1}=2 p_{1} p_{2}+q_{1}^{-\frac{\beta(d+1)+\alpha}{\alpha}} q_{2}^{d}
$$

Taking this Hamiltonian we look for solutions $\mathrm{H}_{2}$ of equations (3.18). We say that a solution of these equations is trivial if the corresponding $V\left(q_{1}, q_{2}\right)$ does not depend on both variables. We do not distinguish solutions which are obtained one from the other by a permutation of pairs of variables $\left(p_{1}, q_{1}\right)$ and $\left(p_{2}, q_{2}\right)$.

Substituting Hamilton function $H_{1}$ of the form (4.2) into the equations (3.18), and taking $\mathrm{H}_{2}$ of the form (3.19) with $M=2$, one can get the following result.

Theorem 4.1. If $\kappa_{2}=1, \alpha \neq 0$ and $\beta \neq 0$ equations (3.18) have only four solutions $V\left(q_{1}, q_{2}\right)$ related to polynomial integrals of motion of degree two in the momenta

\begin{tabular}{|l|l|l|l|l|}
\hline 1 & $\alpha=-\beta$ & $H_{1}=2 p_{1} p_{2}+q_{1}^{d} q_{2}^{d}$ & $I_{2}=q_{1} p_{1}-q_{2} p_{2}$ & $H_{2}=0$ \\
2 & $\alpha=-2 \beta(d+1)$ & $H_{1}=2 p_{1} p_{2}+\frac{q_{2}^{d}}{\sqrt{q_{1}}}$ & $I_{2}=2 p_{1}\left(q_{2} p_{2}-p_{1} q_{1}\right)+\frac{q_{2}^{d+1}}{\sqrt{q_{1}}}$ & $H_{2}=I_{2}^{-1 / \alpha}$ \\
3 & $\alpha=-\frac{\beta(d+1)}{2}$ & $H_{1}=2 p_{1} p_{2}+q_{1} q_{2}^{d}$ & $I_{2}=p_{1}^{2}+\frac{q_{2}^{d+1}}{d+1}$ & $H_{2}=I_{2}^{-1 / 2 \alpha}$ \\
4 & $\alpha=\beta$ & $H_{1}=2 p_{1} p_{2}+\frac{q_{2}^{d}}{q_{1}^{d+2}}$ & $I_{2}=\left(p_{1} q_{1}-p_{2} q_{2}\right)^{2}-\frac{2 q_{2}^{d+1}}{q_{1}^{d+1}}$ & $H_{2}=I_{2} H_{1}^{-1 / 2 \alpha}$ \\
\hline
\end{tabular}

REMARK 4.2 In the case 2 and $3 H_{2}=I_{2}$ satisfies equations (3.18) with $\kappa_{2}=-\alpha$, and $\kappa_{2}=-2 \alpha$, respectively. Similarly, in case $4 H_{2}=I_{2}^{2 \alpha} / H_{1}$ satisfies equations (3.18) with $\kappa_{2}=2 \alpha$.

For each case in the above proposition we suppose that $I_{1}=H_{1}$ and $I_{2}$ are the action variables, and $\omega_{1}, \omega_{2}$ are the corresponding angle variables. Of course, this must be checked case by case. 
In these variables the equations of motion have the form

$$
\dot{I}_{1}=0, \quad \dot{I}_{2}=0, \quad \dot{\omega}_{1}=1 \quad \dot{\omega}_{2}=0 .
$$

Thus, $\omega_{2}$ is a first integral functionally independent with $I_{1}$ and $I_{2}$ [23]. The algebraic relations (3.13), (3.14), and (3.17) allow us to express $\rho$ in term of the action-angle variables. In fact, we have

$$
\begin{array}{lll}
\text { for case 1: } & \left\{\rho, I_{2}\right\}=0, & \rho=I_{2}, \\
\text { for case 2: } & \left\{\rho, I_{2}\right\}=\alpha I_{2}, & \rho=-(\alpha+\beta) I_{1} \omega_{1}-\alpha I_{2} \omega_{2}+F\left(I_{1}, I_{2}\right), \\
\text { for case 3: } & \left\{\rho, I_{2}\right\}=2 \alpha I_{2} & \rho=-(\alpha+\beta) I_{1} \omega_{1}-2 \alpha I_{2} \omega_{2}+F\left(I_{1}, I_{2}\right), \\
\text { for case 4: } & \left\{\rho, I_{2}\right\}=0 & \rho=-(\alpha+\beta) I_{1} \omega_{1}+F\left(I_{1}, I_{2}\right) .
\end{array}
$$

Here angle variables $\omega_{k}$ are defined up to canonical transformations $\omega_{k} \rightarrow \omega_{k}+f\left(I_{k}\right)$, for $k=1,2$.

Later we show that families of systems given by Theorem 4.1 are super-integrable for specific values of parameter $d$. That is, for these values of $d$ the systems admit three functionally independent first integrals $H_{1}, I_{2}$ and $H_{3}$. Some examples of super-integrable systems with additional integral $\mathrm{H}_{3}$ of third, fourth and sixth order in momenta may be found in [23, 24].

According to [23, 24, 25], additional integral of motion $\mathrm{H}_{3}$ is a function on the action variables $I_{1}, I_{2}$ and one angle variable $\omega_{2}$. Below we prove that if we know additional polynomial integral of motion $\mathrm{H}_{3}$, then, using complete algebra of integrals of motion, we can get the angle variable $\omega_{2}$ algebraically.

Some examples of super-integrable systems with additional integral $H_{3}$ of third, fourth and sixth order in momenta may be found in [23, 24].

REMARK 4.3 Usually, see, e.g., [5, 14, 24, 25], in the theory of super-integrable systems we study polynomial Poisson algebra of integrals of motion. In this paper we add some extra generator $\rho$ to this algebra. Let us recall that $\rho$ plays the central role in our bi-Hamiltonian construction (3.8).

\section{Case 1. Radial potential}

In this section we consider Hamiltonian system corresponding to case 1 in Theorem 4.1 and in particular we look for values of $d$ for which the system is super-integrable.

We introduce new canonical variables $\left(r, \varphi, p_{r}, p_{\varphi}\right)$ defined by the following equations

$$
q_{1}=r(\cos \varphi-\mathrm{i} \sin \varphi)=r e^{-\mathrm{i} \varphi}, \quad q_{2}=r(\cos \varphi+\mathrm{i} \sin \varphi)=r e^{\mathrm{i} \varphi},
$$

and

$$
p_{1}=\frac{e^{\mathrm{i} \varphi}}{2}\left(p_{r}+\frac{\mathrm{i}}{r} p_{\varphi}\right), \quad p_{2}=\frac{e^{-\mathrm{i} \varphi}}{2}\left(p_{r}-\frac{\mathrm{i}}{r} p_{\varphi}\right) .
$$

Hamiltonian $H_{1}$ and first integral $J_{2}:=-\mathrm{i} I_{2}$ in new variables read

$$
H_{1}=\frac{p_{r}^{2}}{2}+\frac{p_{\varphi}^{2}}{2 r^{2}}+V(r), \quad V(r)=r^{2 d}, \quad J_{2}=p_{\varphi} .
$$


Thus after this transformation we obtain Hamiltonian of the classical problem of the motion in the field of the radial force written in polar coordinates. In the Cartesian coordinates $x_{1}=r \cos \varphi$, and $x_{2}=r \sin \varphi$, Hamiltonian $H_{1}$ and the first integral $J_{2}$ read

$$
H_{1}=\frac{1}{2}\left(y_{1}^{2}+y_{2}^{2}\right)+r^{2 d}, \quad r^{2}=x_{1}^{2}+x_{2}^{2}, \quad J_{2}=y_{1} x_{2}-y_{2} x_{1},
$$

where $y_{1}$ and $y_{2}$ are the momenta conjugated with $x_{1}$ and $x_{2}$. Thus we obtain a natural Hamiltonian system with the standard form of the kinetic energy and the radial potential. The problem of the existence of one more functionally independent first integral of this system is the question about maximal super-integrability. For natural Hamiltonian systems with homogeneous potentials possessing a proper Darboux point necessary conditions of the maximal super-integrability with first integrals which are meromorphic functions of coordinates and momenta were formulated in [12]. These conditions were obtained from the analysis of invariants of the differential Galois group of variational equations. The considered radial potential possesses infinitely many proper Darboux points and results of [12] can be directly applied. In fact, in Section 3 of this paper Hamiltonian given by $H_{1}$ in (5.4) was considered and the following result was proved.

Theorem 5.1. The radial potential (5.3) is super-integrable iff $d=-1 / 2$ or $d=1$.

Both of distinguished cases are indeed super-integrable with the additional first integrals

$$
H_{3}=\left(p_{1}-p_{2}\right)\left(p_{1} q_{1}-p_{2} q_{2}\right)-\frac{q_{1}+q_{2}}{2 \sqrt{q_{1} q_{2}}}
$$

for $d=-1 / 2$, and

$$
H_{3}=2 p_{2}^{2}+q_{1}^{2}
$$

for $d=1$, respectively. This result reminds us the classical Bertrand theorem [2] which states that the only radial potentials $V(r)=\alpha r^{k}$, for which all bounded orbits are periodic, are those with $k=-1$ and $k=2$. The condition that all bounded orbits of a Hamiltonian system are periodic means that the system is degenerated, i.e., all its invariant tori are one dimensional. Such degeneration appears if the system is maximally super-integrable.

\section{Case 2}

In this section we consider Hamiltonian system corresponding to case 2 in Theorem 4.1. In particular, we distinguish all values of $d$ for which the system is super-integrable. We achieve this thanks to partial separation of variables and a direct integration of equations of motion. In [17] this system is called quasi-separable and in fact we were able to find only one separation relation.

\subsection{Algebraic super-integrability}

One can easy observe that the syzygi exists between $H_{1}$ and $I_{2}$

$$
I_{2}=-2 p_{1}^{2} q_{1}+q_{2} H_{1}
$$


but this is not yet a separation relation. We have to make the change of independent variable $t \rightarrow \tau$ defined by $\mathrm{d} \tau / \mathrm{d} t=1 / \sqrt{q_{1}}$, that transforms our Hamiltonian system into

$$
\begin{array}{ll}
q_{1}^{\prime}=2 p_{2} \sqrt{q_{1}}, & p_{1}^{\prime}=\frac{1}{2} \frac{q_{2}^{d}}{q_{1}}, \\
q_{2}^{\prime}=2 p_{1} \sqrt{q_{1}}, & p_{2}^{\prime}=-d q_{2}^{d-1},
\end{array}
$$

where' denotes differentiation with respect to $\tau$. This transformation changes momenta and now we obtain one separation relation

$$
I_{2}=-\frac{1}{2} q_{2}^{\prime 2}+q_{2} H_{1}
$$

It gives

$$
\int \frac{\mathrm{d} q_{2}}{\sqrt{2\left(q_{2} H_{1}-I_{2}\right)}}=\tau+\beta_{1}
$$

where $\beta_{1}$ is a constant of integration. Here we chosen square root with sign + , that correspond $p_{1}>0$. This integral is elementary and we obtain

$$
H_{1}^{-1} \sqrt{2\left(q_{2} H_{1}-I_{2}\right)}=\tau+\beta_{1} .
$$

In further calculations we choose $\beta_{1}=0$, and then we obtain

$$
q_{2}=\frac{2 I_{2}+H_{1}^{2} \tau^{2}}{2 H_{1}}
$$

Next, we substitute this function into integral $H_{1}$, that is, into equation

$$
H_{1}=\frac{1}{2 q_{1}} \frac{\mathrm{d} q_{1}}{\mathrm{~d} \tau} \frac{\mathrm{d} q_{2}}{\mathrm{~d} \tau}+\frac{q_{2}^{d}}{\sqrt{q_{1}}}
$$

and obtain

$$
\frac{H_{1} \tau}{2 q_{1}} \frac{\mathrm{d} q_{1}}{\mathrm{~d} \tau}+\frac{\left(2 I_{2}+H_{1}^{2} \tau^{2}\right)^{d}}{\left(2 H_{1}\right)^{d} \sqrt{q_{1}}}=H_{1} .
$$

Then, we introduce new dependent variable $v=v(x)$ defined by

$$
q_{1}=\left(\frac{I_{2}}{H_{1}}\right)^{2 d} \frac{v^{2}}{H_{1}^{2}} .
$$

Making this substitution in equation (6.5) and dividing it by $H_{1}$ we obtain

$$
\frac{\tau}{v} \frac{\mathrm{d} v}{\mathrm{~d} \tau}+\frac{\left(2 I_{2}+H_{1}^{2} \tau^{2}\right)^{d}}{\left(2 I_{2}\right)^{d} v}=1
$$

Finally, we make the following change of independent variable

$$
\tau \longmapsto x=-\frac{H_{1}^{2} \tau^{2}}{2 I_{2}}
$$


and from (6.6) we obtain

$$
2 x \frac{\mathrm{d} v}{\mathrm{~d} x}-v+(1-x)^{d}=0 .
$$

This is a non-homogeneous linear equation. Dividing it by $(1-x)^{d}$ and differentiating with respect to $x$ we obtain homogeneous equation of the second order

$$
x(1-x) \frac{\mathrm{d}^{2} v}{\mathrm{~d} x^{2}}+\frac{1-(1-2 d) x}{2} \frac{\mathrm{d} v}{\mathrm{~d} x}-\frac{d}{2} v=0 .
$$

In this equation we recognise immediately Gauss hypergeometric differential equation

$$
x(1-x) w^{\prime \prime}+[c-(a+b+1) x] w^{\prime}-a b w=0,
$$

with parameters

$$
a=-\frac{1}{2}, \quad b=-d, \quad c=\frac{1}{2} .
$$

The general solution of equation (6.8) is following

$$
v(x)=\beta_{2} \sqrt{x}+\beta_{3}{ }_{2} F_{1}\left(-\frac{1}{2},-d, \frac{1}{2}, x\right),
$$

where $\beta_{2}, \beta_{3}$ are constants of integration, and ${ }_{2} F_{1}(a, b, c ; x)$ denotes the hypergeometric function with parameters $a, b$ and $c$. Let us recall that ${ }_{2} F_{1}(a, b, c ; x)$ is a solution of the hypergeometric differential equation (6.9) which is holomorphic at the origin, see Appendix.

Substituting (6.10) into non-homogeneous equation (6.7) we obtain

$$
-\left(\beta_{3}-1\right)(1-x)^{d}=0 .
$$

Hence, $\beta_{3}=1$, and solution of equation (6.7) is given by

$$
v(x)=\beta_{2} \sqrt{x}+{ }_{2} F_{1}\left(-\frac{1}{2},-d, \frac{1}{2}, x\right) .
$$

Next, we return to the original dependent and independent variables and express $\tau$ as a function of $q_{2}$, see (6.4). After this transformation the solution of (6.5) takes the following form

$$
q_{1}=\frac{1}{H_{1}^{2}}\left[\beta_{2} \sqrt{2\left(H_{1} q_{2}-I_{2}\right)}+\left(\frac{I_{2}}{H_{1}}\right)^{d}{ }_{2} F_{1}\left(-\frac{1}{2},-d, \frac{1}{2}, 1-\frac{H_{1}}{I_{2}} q_{2}\right)\right]^{2} .
$$

From the obtained expression one can easily find the following first integral

$$
H_{3}=\sqrt{2} \beta_{2}=\frac{\sqrt{q_{1}} H_{1}-\left(\frac{I_{2}}{H_{1}}\right)^{d}{ }_{2} F_{1}\left(-\frac{1}{2},-d, \frac{1}{2}, 1-\frac{H_{1}}{I_{2}} q_{2}\right)}{\sqrt{q_{2} H_{1}-I_{2}}} .
$$

We prove the following theorem. 
Theorem 6.1. Hamiltonian system given by

$$
H=2 p_{1} p_{2}+\frac{q_{2}^{d}}{\sqrt{q_{1}}}
$$

is super-integrable with algebraic additional first integral (6.14) iff $d=p$, or $d=-(2 p-1) / 2$, for a positive integer $p$.

Proof. The first integral (6.14) is algebraic iff the hypergeometric function ${ }_{2} F_{1}$ appearing in its definition is algebraic. This implies that the hypergeometric equation (6.9) has an algebraic solution holomorphic at the origin.

For equation (6.9) the differences of exponents at singularities are

$$
\lambda=1-c=\frac{1}{2}, \quad v=c-a-b=d+1, \quad \mu=b-a=-d+\frac{1}{2} .
$$

This is why, by Lemma A.1, see Appendix, the Gauss hypergeometric equation (6.9) is reducible. In fact, for an arbitrary value of $d$ we have

$$
\lambda+\mu+v=2, \quad-\lambda+\mu+v=1, \quad \lambda-\mu+v=2 d+1, \quad \lambda+\mu-v=-2 d .
$$

Thus, always at least one of this expressions is odd integer, and this proves our claim.

As equation (6.9) is reducible its one solution is algebraic. In fact $w_{1}=\sqrt{x}$ is a solution of this equation. As we remarked, if first integral (6.14) is algebraic, then equation (6.9) has a solution $w_{2}$ which is algebraic and holomorphic at the origin. Obviously $w_{2}$ is linearly independent with $w_{1}$. In effect all solutions of equation (6.9) are algebraic.

Now we apply Theorem A.3 in order to find values of $d$ for which equation (6.9) has only algebraic solutions. The first assumption of this theorem is that all exponents at all singularities are rational. For equation (6.9) exponents at $x=0, x=1$, and $x=\infty$ are given by

$$
\left\{0, \frac{1}{2}\right\}, \quad\{0, d+1\}, \quad\left\{-\frac{1}{2},-d\right\} .
$$

Thus, if all solutions of equation (6.9) are algebraic, then $d$ must be rational.

The next assumption of the theorem is that among four numbers (6.16) exactly two or four are odd integers. This condition implies that $d=p / 2$, with $p \in \mathbb{Z}$.

Now we have to check the presence of logarithms in local solutions of (6.9) around particular singularities. At first we assume that $p$ is even i.e. $p=2 q$ and $d=q \in \mathbb{Z}$. In this case the difference of exponents is integer only for singularity $x=1$. For $q \in \mathbb{N}$, the difference of exponents is $m=q+1-0=q+1$. Hence, according to Appendix, the set $\langle m\rangle$ is $\langle m\rangle=\{1,2, \ldots, q+1\}$, see (A.5). Now we have to check if

$$
e_{1,1}+e_{0, i}+e_{\infty, j} \notin\langle m\rangle, \quad \text { for } \quad i, j \in\{1,2\},
$$

with $e_{1,1}=q+1$. But we see that $q+1+0-q=1 \in\langle m\rangle$. Thus for $d=p / 2=q$ positive integer solutions of (6.9) contain no logarithms. For $q$ negative integer difference of exponents is $m=0-(q+1)=-q-1$, so $\langle m\rangle=\{1,2, \ldots,-q-1\}$. Now we have to check all conditions (6.17) with $e_{1,1}=0$. We obtain successively $0+0-1 / 2=-1 / 2 \notin\langle m\rangle$, $0+0-q=-q \notin\langle m\rangle, 0+1 / 2-1 / 2=0 \notin\langle m\rangle$, and $0+1 / 2-q=-q+1 / 2 \notin\langle m\rangle$. This 
means that if $q$ is a negative integer, then logarithmic terms appear in solutions of (6.9). Similar considerations for $p=2 q-1$ show that logarithmic terms appear in local solutions of (6.9) around $x=\infty$ iff $d=p / 2=(2 q-1) / 2$, with positive integer $q$.

When hypergeometric function ${ }_{2} F_{1}$ is an algebraic function of its argument, then $\mathrm{H}_{3}$ defined in (6.14) is an algebraic function.

Finally, one can check by direct substitution that for $d=0$ hypergeometric function with the above parameters is equal to 1 , and this ends our proof.

\subsection{Additional integrals polynomial in momenta}

Let us notice that in general the first integral given by (6.14) for values of $d$ specified in Theorem 6.1 is not polynomial in the momenta. However, we can show the following.

Lemma 6.2. If $d=p \in \mathbb{N}$, then

$$
\widetilde{H}_{3}=\widetilde{H}_{3, p}:=H_{3} H_{1}^{p},
$$

with $\mathrm{H}_{3}$ given by (6.14) is a first integral of the system generated by Hamiltonian

$$
H=H_{1}=2 p_{1} p_{2}+\frac{q_{2}^{p}}{\sqrt{q_{1}}}
$$

Moreover, $\widetilde{H}_{3}$ is a polynomial in momenta and has degree $2 p+1$.

Proof. Clearly $\widetilde{H}_{3}$ is a first integral of $H$. In order to show that $\widetilde{H}_{3}$ is a polynomial in momenta we notice that

$$
\widetilde{H}_{3}=H_{3} H_{1}^{p}=\frac{1}{\sqrt{2 q_{1}} p_{1}}\left[\sqrt{q_{1}} H_{1}^{p+1}-I_{2}^{p} W_{p}\left(\frac{-2 p_{1}^{2} q_{1}}{I_{2}}\right)\right],
$$

where

$$
W_{p}(x):={ }_{2} F_{1}\left(-\frac{1}{2},-p, \frac{1}{2}, x\right), \quad W_{p}(0)=1,
$$

is a polynomial of degree $p$, see Appendix. Thus,

$$
B:=\sqrt{q_{1}} H_{1}^{p+1}-I_{2}^{p} W_{p}\left(\frac{-2 p_{1}^{2} q_{1}}{I_{2}}\right)
$$

is a polynomial in momenta of degree $2 p+2$. Moreover we have

$$
\sqrt{q_{1}} H_{1}^{p+1}=\frac{q_{2}^{p(p+1)}}{q_{1}^{p / 2}}+\cdots, \quad \text { and } \quad I_{2}^{p} W_{p}\left(\frac{-2 p_{1}^{2} q_{1}}{I_{2}}\right)=\frac{q_{2}^{p(p+1)}}{q_{1}^{p / 2}}+\cdots,
$$

where dots denote terms of degree at lest one in $p_{1}$. This shows that $B$ is divisible by $p_{1}$. In effect $\widetilde{H}_{3}$ is polynomial in the momenta and has degree $2 p+1$.

Lemma 6.3. If $d=-(2 p-1) / 2$ for a certain $p \in \mathbb{N}$, then

$$
\widetilde{H}_{3}=\widetilde{H}_{3, p}:=H_{3} I_{2}^{p} / H_{1},
$$


with $\mathrm{H}_{3}$ and $\mathrm{I}_{2}$ given by (6.14) and (6.1) is a first integral of system generated by Hamiltonian

$$
H=H_{1}=2 p_{1} p_{2}+\frac{q_{2}^{p}}{\sqrt{q_{1}}} .
$$

Moreover $\widetilde{H}_{3}$ is a polynomial in momenta and has degree $2 p-1$.

Proof. First we show that for the assumed values of $d$ we have

$$
{ }_{2} F_{1}\left(-\frac{1}{2},-d, \frac{1}{2}, x\right)={ }_{2} F_{1}\left(-\frac{1}{2}, \frac{1}{2}(2 p-1), \frac{1}{2}, x\right)=(1-x)^{-(2 p-3) / 2} W_{p-1}(x),
$$

where $W_{p-1}(x)$ is a polynomial of degree $p-1$, and $W_{p-1}(0)=1$.

In fact, for an arbitrary $p$, hypergeometric equation (6.9) has in a neighbourhood of $x=1$ a local solution of the form $w=(1-x)^{-(2 p-3) / 2} u(x)$ where $u(x)$ is a function holomorphic at the origin and $u(0)=1$. This is so, because $-(2 p-3) / 2$ is an exponent at $x=1$ for this equation. In order to find $u(x)$ we make substitution $w=(1-x)^{-(2 p-3) / 2} u(x)$ in equation (6.9). The obtained equation is again a hypergeometric equation. Its solution holomorphic at the origin is

$$
u(x)={ }_{2} F_{1}\left(1,1-p, \frac{1}{2}, x\right) .
$$

As $p$ is a positive integer, $u(x)$ is a polynomial of degree $p-1$.

Now, we have

$$
\widetilde{H}_{3}:=H_{3} I_{2}^{p} / H_{1}=\frac{1}{\sqrt{2 q_{1}} p_{1}}\left[\sqrt{q_{1}} I_{2}^{p}-q_{2}^{-(2 p-3) / 2} I_{2}^{p-1} W_{p-1}\left(-\frac{p_{1}^{2} q_{1}}{I_{2}}\right)\right] .
$$

As in the proof of the previous lemma it is easy to notice that expression in the square bracket is a polynomial in momenta which is divisible by $p_{1}$, and, as a result, $\widetilde{H}_{3}$ is a polynomial with respect to the momenta of degree $2 p-1$.

\subsection{Examples}

Here we consider two examples of super-integrable systems given by Theorem 6.1. Let us take $d=p=2$. In this case the Hamiltonian function has the form

$$
H=H_{1}=2 p_{1} p_{2}+\frac{q_{2}^{2}}{\sqrt{q_{1}}} .
$$

Then, according Theorem 4.1 first integral $I_{2}$ is following

$$
I_{2}=2 p_{1}\left(p_{2} q_{2}-p_{1} q_{1}\right)+\frac{q_{2}^{3}}{\sqrt{q_{1}}} .
$$

The second additional first integral $\widetilde{H}_{3}=\widetilde{H}_{3, p}$ which guarantees the super-integrability is given by (6.18). For $p=2$, it has, up to a multiplicative constant, the following form

$$
\widetilde{H}_{3}=q_{1}^{-1}\left[-16 p_{1}^{3} q_{1}^{5 / 2}+3 p_{2} q_{2}^{4}+12 p_{1}^{2} p_{2} q_{1}\left(p_{2}^{2}+2 \sqrt{q_{1}} q_{2}\right)+12 p_{1}\left(p_{2}^{2} \sqrt{q_{1}} q_{2}^{2}+q_{1} q_{2}^{3}\right)\right] .
$$


Moreover, there exists other first integral $\mathrm{H}_{4}$ of the form

$$
H_{4}=-18 p_{1} p_{2}^{3}+q_{1}^{-1 / 2}\left[48 p_{1}^{2} q_{1}^{2}-72 p_{1} p_{2} q_{1} q_{2}-9 p_{2}^{2} q_{2}^{2}\right]-32 q_{2}^{3} .
$$

It is functionally independent with $H_{1}$ and $I_{2}$. Integrals $H_{1}, I_{2}, \widetilde{H}_{3}$ and $H_{4}$ are algebraically dependent as we have the following identity

$$
H_{1}^{3} H_{4}+\widetilde{H}_{3}^{2}+32 I_{2}^{3}=0 .
$$

We note that $I_{2}^{-1 / \alpha}, \widetilde{H}_{3}^{-2 /(3 \alpha)}, H_{4}^{-2 / \alpha}$ satisfy system (3.18) with normalisation $\kappa_{2}=1$.

Examples with other values of $p$ allow us to formulate the following.

Conjecture 6.4. In the case 2 of Theorem 4.1 if $d=p \in \mathbb{N}$, then there exists $\alpha_{p} \in \mathbb{R}$, such that first integral $\mathrm{H}_{4}$ defined by the following relation

$$
H_{1}^{p+1} H_{4}+\widetilde{H}_{3, p}^{2}+\alpha_{p} I_{2}^{p+1}=0,
$$

is polynomial in the momenta of degree $2 p$. Moreover, integrals $H_{1}, I_{2}$ and $H_{4}$ are functionally independent.

Now, we consider the element of family with $d=-(2 p-1) / 2$ for $p=3$. Then Hamilton is

$$
H_{1}=H=2 p_{1} p_{2}+\frac{1}{\sqrt{q_{1}} q_{2}^{5 / 2}}
$$

and first integral $I_{2}$ takes the form

$$
I_{2}=2 p_{1}\left(p_{2} q_{2}-p_{1} q_{1}\right)+\frac{1}{\sqrt{q_{1}} q_{2}^{3 / 2}} .
$$

The explicit form of additional first integral $\widetilde{H}_{3}=\widetilde{H}_{3, p}$ defined by (6.19) is, up to a multiplicative constant, equal to

$$
\widetilde{H}_{3}=4 p_{1}^{2}\left(p_{1} q_{1}-p_{2} q_{2}\right)^{3}-\frac{4 p_{1}\left(5 p_{1}^{2} q_{1}^{2}-12 p_{1} p_{2} q_{1} q_{2}+3 p_{2}^{2} q_{2}^{2}\right)}{3 \sqrt{q_{1}} q_{2}^{3 / 2}}+\frac{5 p_{1} q_{1}-p_{2} q_{2}}{q_{1} q_{2}^{3}} .
$$

One can check that

$$
H_{4}=18 p_{1}\left(p_{1} q_{1}-p_{2} q_{2}\right)^{3}+\frac{-33 p_{1}^{2} q_{1}^{2}+90 p_{1} p_{2} q_{1} q_{2}-9 p_{2}^{2} q_{2}^{2}}{\sqrt{q_{1} q_{2}^{3 / 2}}}+\frac{32}{q_{2}^{3}},
$$

is also a first integral of degree $2 p-2=4$ with respect to the momenta, and that the following relation is satisfied

$$
32 H_{1}^{3}-9 \widetilde{H}_{3}^{2}-I_{2}^{3} H_{4}=0 .
$$

Moreover, $I_{2}^{-1 / \alpha}, \widetilde{H}_{3}^{-1 /(2 \alpha)}, H_{4}^{-1 / \alpha}$ satisfy system (3.18) with normalisation $\kappa_{2}=1$.

As in the previous case, additional examples justify the following conjecture.

Conjecture 6.5. In the case 2 of Theorem 4.1. if $d=-(2 p-1) / 2$ with $p \in \mathbb{N}$, then there exists $\alpha_{p} \in \mathbb{R}$, such that first integral $\mathrm{H}_{4}$ defined by the following relation

$$
H_{1}^{p}+\alpha_{p} \widetilde{H}_{3, p}^{2}-I_{2}^{p} H_{4}=0,
$$

is polynomial in the momenta of degree $2 p-2$. Moreover, integrals $H_{1}, I_{2}$ and $H_{4}$ are functionally independent. 


\section{Case 3. Super-integrability without additive separation of vari- ables}

In case 3 of Theorem 4.1 we have

$$
\beta=-\frac{2 \alpha}{d+1}, \quad \rho=\alpha\left(p_{1} q_{1}-\frac{2 p_{2} q_{2}}{d+1}\right), \quad H_{1}=2 p_{1} p_{2}+q_{1} q_{2}^{d}
$$

and one solution of the equations (3.18) is equal to

$$
H_{2}=I_{2}^{-1 / 2 \alpha}, \quad I_{2}=p_{1}^{2}+\kappa q_{2}^{d+1}, \quad \kappa=\frac{1}{d+1} .
$$

As in the previous section our aim is to distinguish cases when the system is superintegrable.

\subsection{Algebraic super-integrability}

In order to find the second additional first integral $\mathrm{H}_{3}$ we proceed as follows. From the integral $I_{2}$ (7.2) we directly obtain the first quadrature

$$
\beta_{1}+t=-\int^{q_{2}} \frac{\mathrm{d} x}{2 \sqrt{I_{2}-\kappa x^{d+1}}}
$$

After calculation of this quadrature we have to substitute solution $q_{2}=q_{2}\left(t, \beta_{1}\right)$ into the Hamiltonian

$$
I_{1}=\frac{1}{2} \frac{\mathrm{d} q_{1}}{\mathrm{~d} t} \frac{\mathrm{d} q_{2}}{\mathrm{~d} t}+q_{1} q_{2}^{d}
$$

and solve the obtained quadrature with respect to $q_{1}$. However to find $q_{2}=q_{2}(t)$ we have to invert explicitly quadrature (7.3), and it is unclear how to do this. By this reason we proceed in a different way. Using function $\rho$ and algebraic relations (4.3) we can get the second angle variable

$$
\omega_{2}=\frac{\rho}{2 \alpha I_{2}}+\frac{d-1}{d+1} \frac{I_{1}}{2 I_{2}} \int^{q_{2}} \frac{\mathrm{d} x}{2 \sqrt{I_{2}-\kappa x^{d+1}}}
$$

In generic case $\omega_{2}$ is the multi-valued function on the whole phase space [23, 24, 25].

We want to distinguish values of $d$ for that system in case 3 from Theorem 4.1 governed by Hamiltonian $H_{1}$ given by (7.1) is super-integrable. Since $H_{1}=I_{1}$, thus $\omega_{2}$ is a first integral. So, the following function

$$
H_{3}=4 \alpha(d+1) I_{2} \omega_{2}=2(d+1) \rho+(d-1) \alpha I_{1} \int^{q_{2}} \frac{\mathrm{d} x}{\sqrt{I_{2}-\kappa x^{d+1}}} .
$$

is also a first integral which we are going to analyse in details.

In the integral presented in $\mathrm{H}_{3}$ we make the following substitution

$$
y=\frac{\kappa}{I_{2}} x^{d+1}
$$


which gives

$$
\int \frac{\mathrm{d} x}{\sqrt{I_{2}-\kappa x^{d+1}}}=\frac{1}{(d+1) \sqrt{I_{2}}}\left(\frac{I_{2}}{\kappa}\right)^{\frac{1}{d+1}} \int \frac{y^{-\frac{d}{d+1}} \mathrm{~d} y}{\sqrt{1-y}} .
$$

Let us denote

$$
\int \frac{y^{-\frac{d}{d+1}} \mathrm{~d} y}{\sqrt{1-y}}=(d+1) y^{\frac{1}{d+1}} v(y)
$$

where $v(y)$ is a certain function of $y$. Differentiating both sides of this equation with respect to $y$, after obvious simplifications we obtain the following linear non-homogeneous equation for the unknown function $v(y)$

$$
\frac{1}{\sqrt{1-y}}=v+(d+1) y v^{\prime}
$$

Multiplying it by $\sqrt{1-y}$ and differentiating once more we obtain

$$
y(1-y) v^{\prime \prime}+\left(\frac{d+2}{d+1}-\frac{3 d+5}{2 d+2} y\right) v^{\prime}-\frac{1}{2 d+2} v=0 .
$$

This is the Gauss hypergeometric equation with parameters

$$
a=\frac{1}{2}, \quad b=\frac{1}{d+1}, \quad c=1+\frac{1}{d+1} .
$$

General solution of (7.7) has the form

$$
v(y)=\beta_{2} y^{-1 /(d+1)}+\beta_{3} F_{1}\left(\frac{1}{2}, \frac{1}{d+1}, 1+\frac{1}{d+1}, y\right) .
$$

Substituting it into equation (7.6) we find out that it is its solution iff $\beta_{3}=1$. Without loss of the generality we can assume that $\beta_{2}=0$, and then in variable $x$ considered integral is

$$
\int \frac{\mathrm{d} x}{\sqrt{I_{2}-\kappa x^{d+1}}}=\frac{x}{\sqrt{I_{2}}}{ }_{2} F_{1}\left(\frac{1}{2}, \frac{1}{d+1}, 1+\frac{1}{d+1}, \frac{\kappa x^{d+1}}{I_{2}}\right) .
$$

Thus our first integral takes the final form

$$
H_{3}=2(d+1) \rho+(d-1) \alpha \frac{I_{1} q_{2}}{\sqrt{I_{2}}}{ }_{2} F_{1}\left(\frac{1}{2}, \frac{1}{d+1}, 1+\frac{1}{d+1}, \frac{\kappa q_{2}^{d+1}}{I_{2}}\right) .
$$

This first integral is functionally independent with $I_{1}$ and $I_{2}$. In general hypergeometric functions are not single-valued with sinularities of non-algebraic character. Thus, in order to obtain first integrals with "good" behaviour, we will restrict to cases when function ${ }_{2} F_{1}$ is algebraic. In other words, we are looking for such values of $d$ that equation (7.7) has a solution that is algebraic. At first let us note that by Lemma A.1 equation (7.7) is reducible for any value of $d$. Indeed, the differences of exponents at singularities of equation (7.7) are

$$
\lambda=1-c=-\frac{1}{d+1}, \quad v=c-a-b=\frac{1}{2}, \quad \mu=b-a=\frac{1}{d+1}-\frac{1}{2},
$$


and among numbers

$$
\lambda+\mu+v=0, \quad-\lambda+\mu+v=\frac{2}{d+1}, \quad \lambda-\mu+v=1-\frac{2}{d+1}, \quad \lambda+\mu-v=-1,
$$

we have one even and one odd number. This means that we can use Theorem A.3 in order to find values of $d$ for which equation (7.7) has only algebraic solutions.

Theorem 7.1. Hamiltonian system given by

$$
H=2 p_{1} p_{2}+q_{1} q_{2}^{d}
$$

is super-integrable with algebraic additional first integral (7.9) iff $d$ takes the form

$$
d=\frac{1-p}{p}, \quad \text { or } \quad d=\frac{1+2 p}{1-2 p}
$$

for $p \in \mathbb{N}$.

Proof. We apply directly Theorem A.3. Exponents at singularities $x=0, x=1$ and $x=\infty$ for equation (7.7) are

$$
\left\{0,-\frac{1}{d+1}\right\}, \quad\left\{0, \frac{1}{2}\right\}, \quad\left\{\frac{1}{2}, \frac{1}{d+1}\right\},
$$

respectively. The first condition of Theorem A.3 implies that $d$ has to be rational. The second condition can be fulfilled only when two among number (7.11) are odd, and this implies that

$$
\frac{2}{d+1}=r, \quad \text { so } \quad d=\frac{2-r}{r}
$$

for a certain $r \in \mathbb{Z}$.

Now we have to check the presence of logarithms in local solutions of (7.7) around particular singularities. In this aim we apply Lemma A.2. For $d$ given by (7.13) exponents are following

$$
\left\{0,-\frac{r}{2}\right\}, \quad\left\{0, \frac{1}{2}\right\}, \quad\left\{\frac{1}{2}, \frac{r}{2}\right\} \text {. }
$$

We see that differences of exponents can be integer positive only for $x=0$ or $x=\infty$ depending on the parity of $r$ as well as the positive or negative sign of $r$. An analysis similar to that done in the proof of Theorem 6.1 shows that logarithmic terms appear in local solutions iff either $r$ is positive and odd, or $r$ is negative and even.

If all solutions of the considered equation are algebraic then, by Theorem A.3 none of local solution has a logarithmic term. Thus, by the above considerations, either $r$ is a positive and even integer, or it is a negative and odd integer. Substituting $r=2 p$ as well as $r=-(2 p-1)$ with $p \in \mathbb{N}$ into (7.13) we obtain (7.12), and this ends the proof. 


\subsection{Additional first integrals polynomial in the momenta}

If assumptions of Theorem 7.1 are fulfilled, then first integral given by (7.9) is algebraic but it is not polynomial with respect to the momenta. However we can find such integrals using the following.

Lemma 7.2. If

$$
d=\frac{1-p}{p} \text { for a certain } p \in \mathbb{N},
$$

then $\mathrm{H}_{3}$ given by (7.9) can be uniquely written in the following form

$$
H_{3}:=\widetilde{H}_{3, p}+\sqrt{I_{2}} J_{3, p},
$$

where $\widetilde{H}_{3, p}$ and $J_{3, p}$ are first integrals polynomial in the momenta of the system with Hamiltonian $H=H_{1}$ defined by (7.1). Moreover, $\widetilde{H}_{3, p}$ has degree $2 p-1$ with respect to the moment and is functionally independent together with $\mathrm{H}$ and $\mathrm{I}_{2}$.

Proof. For the specified form of $d$, we have

$$
F(x):={ }_{2} F_{1}\left(\frac{1}{2}, \frac{1}{d+1}, 1+\frac{1}{d+1}, x\right)={ }_{2} F_{1}\left(\frac{1}{2}, p, 1+p, x\right) .
$$

We show that

$$
F(x)=x^{-p}\left(C+\sqrt{1-x} W_{p-1}(x)\right),
$$

where $W_{p-1}(x)={ }_{2} F_{1}\left(\frac{1}{2}, 1-p, 1-p, x\right)$ is a polynomial of degree $p-1$, and

$$
C={ }_{2} F_{1}\left(\frac{1}{2}, p, 1+p, 1\right)=\sqrt{\pi} \frac{\Gamma(1+p)}{\Gamma\left(\frac{1}{2}+p\right)}=\frac{2^{p} p !}{(2 p-1) ! !} .
$$

In fact, $v:=F(x)$ is a solution of hypergeometric equation (7.7) holomorphic at the origin. Making substitution (7.16) we find that $W_{p-1}(x)$ coincides with ${ }_{2} F_{1}\left(\frac{1}{2}, 1-p, 1-p, x\right)$. As $p$ is a positive integer $W_{p-1}(x)$ is a polynomial of degree $p-1$.

In the considered case we have

$$
x:=\frac{q_{2}^{d+1}}{(d+1) I_{2}}=\frac{p q_{2}^{1 / p}}{I_{2}}, \quad \text { and } \quad 1-x=\frac{p_{1}^{2}}{I_{2}} .
$$

Thus we can rewrite $\mathrm{H}_{3}$ given by (7.9) in the following form

$$
H_{3}=\frac{2}{p} \rho+\alpha \frac{1-2 p}{p^{p+1}} I_{1} \sqrt{I_{2}} I_{2}^{p-1}\left(C+\frac{p_{1}}{\sqrt{I_{2}}} W_{p-1}\left(\frac{p q_{2}^{1 / p}}{I_{2}}\right)\right),
$$

i.e., it has the form (7.14) with

$$
\widetilde{H}_{3, p}:=\alpha \frac{2}{p}\left(p_{1} q_{1}-2 p p_{2} q_{2}\right)+\alpha \frac{1-2 p}{p^{p+1}} p_{1} I_{1} I_{2}^{p-1} W_{p-1}\left(\frac{p q_{2}^{1 / p}}{I_{2}}\right),
$$

and

$$
J_{3, p}:=\alpha C \frac{1-2 p}{p^{p+1}} I_{1} I_{2}^{p-1} .
$$

Clearly, these are polynomials with respect to momenta, and $\widetilde{H}_{3, p}$ has degree $2 p-1$. 
Lemma 7.3. If

$$
d=\frac{1+2 p}{1-2 p} \quad \text { for a certain } \quad p \in \mathbb{N},
$$

then

$$
\widetilde{H}_{3, p}:=H_{3} I_{2}^{p},
$$

where $\mathrm{H}_{3}$ given by (7.9) is a first integral polynomial in the momenta of degree $2 p+1$.

Proof. We show that if $d$ is given by (7.20), then

$$
{ }_{2} F_{1}\left(\frac{1}{2}, \frac{1}{d+1}, 1+\frac{1}{d+1}, y\right)={ }_{2} F_{1}\left(\frac{1}{2}, \frac{1}{2}-p, \frac{3}{2}-p, y\right)=\sqrt{1-y} R_{p-1}(y),
$$

where $R_{p-1}(y)$ is a polynomial of degree $p-1$.

Recall that hypergeometric function given above is a solution of equation (7.7). If we make substitution

$$
v(y)=\sqrt{1-y} u(y)
$$

in equation (7.7), then we obtain again the Gauss hypergeometric equation

$$
y(1-y) u^{\prime \prime}+\left(\frac{3}{2}-p+(p-3) y\right) u^{\prime}+(p-1) u=0,
$$

with the parameters

$$
a=1-p, \quad b=1, \quad c=\frac{1}{2}(3-2 p) .
$$

For $p \in \mathbb{N}$, parameter $a$ is non-positive integer, thus function $u(y):={ }_{2} F_{1}(a, b, c, y)$ is a polynomial of degree $p-1$. Hence, we have

$$
\begin{aligned}
v(y)={ }_{2} F_{1}\left(\frac{1}{2}, \frac{1}{2}-p, \frac{3}{2}-p, y\right)=\sqrt{1-y} u(y) & = \\
& \sqrt{1-y}{ }_{2} F_{1}\left(1-p, 1, \frac{1}{2}(3-2 p), y\right) .
\end{aligned}
$$

It follows that $R_{p-1}(y)$ is equal to ${ }_{2} F_{1}\left(1-p, 1, \frac{1}{2}(3-2 p), y\right)$, and so it is a polynomial of degree $p-1$, as we claimed.

Now we have to show that $\widetilde{H}_{3, p}$ given by (7.21) is a polynomial with respect to the momenta. But we can write

$$
\begin{array}{r}
\widetilde{H}_{3, p}=\alpha I_{2}^{p}\left(2(d+1) q_{1} p_{1}-q_{2} p_{2}\right)+\alpha(d-1) I_{2}^{p} \frac{I_{1} q_{2}}{\sqrt{I_{2}}} \sqrt{1-\frac{\kappa q_{2}^{d+1}}{I_{2}}} R_{p-1}\left(\frac{\kappa q_{2}^{d+1}}{I_{2}}\right) \\
=\alpha I_{2}^{p}\left(2(d+1) q_{1} p_{1}-q_{2} p_{2}\right)+\alpha(d-1) I_{1} q_{2} \sqrt{I_{2}} \sqrt{1-\frac{\kappa q_{2}^{d+1}}{I_{2}}} S_{p-1},
\end{array}
$$

where

$$
S_{p-1}=I_{2}^{p-1} R_{p-1}\left(\frac{\kappa q_{2}^{d+1}}{I_{2}},\right)
$$


is a polynomial of degree not higher than $2(p-1)$ with respect to the momenta. Now, it is enough to notice that according to (7.3) and (7.2), we have

$$
\sqrt{I_{2}} \sqrt{1-\frac{\kappa q_{2}^{d+1}}{I_{2}}}=-p_{1}
$$

in order to see that both terms in (7.27) are polynomial with respect to the momenta. Moreover, it is easy to see that the degree of $\widetilde{H}_{3, p}$ with respect to the momenta is $2 p+1$.

\subsection{Examples}

As example we take the family with $d=(1-p) / p$, and we choose $p=5$. In this case we have

$$
H_{1}=2 p_{1} p_{2}+\frac{q_{1}}{q_{2}^{4 / 5}}, \quad I_{2}=p_{1}^{2}+5 q_{2}^{1 / 5} .
$$

Up to a multiplicative constant, the first integral $\widetilde{H}_{3}:=\widetilde{H}_{3, p}$ defined in Lemma 7.2 by equation (7.14) has the following form

$$
\begin{aligned}
\widetilde{H}_{3}= & q_{2}^{-4 / 5}\left[128 p_{1}^{8} p_{2} q_{2}^{4 / 5}+64 p_{1}^{6}\left(p_{1} q_{1}+35 p_{2} q_{2}\right)+560 p_{1}^{4} q_{2}^{1 / 5}\left(2 p_{1} q_{1}+25 p_{2} q_{2}\right)\right. \\
& \left.+7000 p_{1}^{2} q_{2}^{2 / 5}\left(p_{1} q_{1}+5 p_{2} q_{2}\right)+4375 q_{2}^{3 / 5}\left(4 p_{1} q_{1}+5 p_{2} q_{2}\right)\right] .
\end{aligned}
$$

There exists also other first integral of degree $2 p-2=8$ in the momenta

$$
\begin{aligned}
H_{4}= & 896 p_{1}^{6} p_{2}\left(p_{1} q_{1}-p_{2} q_{2}\right)+224 q_{2}^{-4 / 5} p_{1}^{4}\left(2 p_{1}^{2} q_{1}^{2}+66 p_{1} p_{2} q_{1} q_{2}-65 p_{2}^{2} q_{2}^{2}\right) \\
& +16 q_{2}^{-3 / 5} p_{1}^{2}\left(476 p_{1}^{2} q_{1}^{2}+5215 p_{1} p_{2} q_{1} q_{2}-5025 p_{2}^{2} q_{2}^{2}\right) \\
& +5 q_{2}^{-2 / 5}\left(9072 p_{1}^{2} q_{1}^{2}+32920 p_{1} p_{2} q_{1} q_{2}-30625 p_{2}^{2} q_{2}^{2}\right)+102400 q_{1}^{2} q_{2}^{-1 / 5},
\end{aligned}
$$

and the following relation holds

$$
3125 H_{4}+\widetilde{H}_{3}^{2}-4096 H_{1}^{2} I_{2}^{7}=0 .
$$

Moreover, $I_{2}^{-1 /(2 \alpha)}, \widetilde{H}_{3}^{1 /(2 \alpha)}, H_{4}^{1 /(4 \alpha)}$ satisfy system (3.18) with normalisation $\kappa_{2}=1$. Other examples justify the following.

Conjecture 7.4. In the case 3 of Theorem 4.1 if $d=(1-p) / p$ for a certain $p \in \mathbb{N}$, then there exists $\alpha_{p} \in \mathbb{R}$, such that first integral $H_{4}$ given by

$$
H_{4}=\widetilde{H}_{3, p}^{2}+\alpha_{p} I_{2}^{2 p-3} H_{1}^{2}
$$

is polynomial in the momenta of degree $2 p-2$. Moreover, integrals $H_{1}, I_{2}$ and $H_{4}$ are functionally independent.

Now, let $d=(1+2 p) /(1-2 p)$, and we consider the case with $p=2$, i.e., $d=-5 / 3$. In this case we have

$$
H_{1}=2 p_{1} p_{2}+\frac{q_{1}}{q_{2}^{5 / 3}}, \quad \text { and } \quad I_{2}=p_{1}^{2}-\frac{3}{2 q_{2}^{2 / 3}} .
$$


The additional first integral $\widetilde{H}_{3}:=\widetilde{H}_{3, p}$ defined by (7.21) of degree $2 p+1$ with respect to the momenta is, up to a multiplicative constant, of the following form

$$
\widetilde{H}_{3}=4 p_{1}^{4}\left(p_{2} q_{2}-p_{1} q_{1}\right)+\frac{4 p_{1}^{2}\left(5 p_{1} q_{1}-9 p_{2} q_{2}\right)}{q_{2}^{2 / 3}}-\frac{9\left(5 p_{1} q_{1}+3 p_{2} q_{2}\right)}{q_{2}^{4 / 3}} .
$$

We have also first integral $H_{4}$ of degree $2 p$ with respect to the momenta given by

$$
H_{4}=p_{1}^{2}\left(p_{1} q_{1}-p_{2} q_{2}\right)^{2}-\frac{\left(11 p_{1} q_{1}-27 p_{2} q_{2}\right)\left(p_{1} q_{1}-p_{2} q_{2}\right)}{2 q_{2}^{2 / 3}}+\frac{16 q_{1}^{2}}{q_{2}^{4 / 3}}
$$

and the following identity holds

$$
864 H_{1}^{2}-\widetilde{H}_{3}^{2}+16 H_{4} I_{2}^{3}=0
$$

Moreover, $I_{2}^{-1 /(2 \alpha)}, \widetilde{H}_{3}^{-1 /(4 \alpha)}, H_{4}^{-1 /(2 \alpha)}$ satisfy system (3.18) with normalisation $\kappa_{2}=1$.

Other examples with different values of $p$ allow us to formulate the following.

Conjecture 7.5. In the case 3 of Theorem 4.1 if $d=(1-2 p) /(1+2 p)$ for a certain $p \in \mathbb{N}$, then there exists $\alpha_{p} \in \mathbb{R}$, such that first integral $\mathrm{H}_{4}$ defined by

$$
H_{4} I_{2}^{p+1}+\widetilde{H}_{3, p}^{2}+\alpha_{p} H_{1}^{2}=0
$$

is polynomial in the momenta of degree $2 p$. Moreover, integrals $H_{1}, I_{2}$ and $H_{4}$ are functionally independent.

Let us determine the complete algebra generated by first integrals (7.29), (7.30), (7.31) and $\rho$ which in the case $d=-5 / 3$ has the following form

$$
\rho=-\frac{1}{2}\left(q_{1} p_{1}+3 q_{2} p_{2}\right) .
$$

It is easy to check that first integral given by

$$
\widetilde{H}_{3}=\left\{I_{2}, H_{4}\right\},
$$

satisfies equations (3.18) with $\kappa_{2}=2$. The complete algebra of integrals and $\rho$ is given by

$$
\begin{array}{lll}
\left\{I_{1}, \rho\right\}=2 I_{1}, \quad\left\{I_{2}, \rho\right\}=I_{2}, & \left\{H_{4}, \rho\right\}=H_{4}, \quad\left\{\widetilde{H}_{3}, \rho\right\}=2 \widetilde{H}_{3}, \quad\left\{I_{2}, H_{4}\right\}=\widetilde{H}_{3}, \\
\left\{I_{1}, I_{2}\right\}=\left\{I_{1}, \widetilde{H}_{3}\right\}=\left\{I_{1}, H_{4}\right\}=0, & \left\{I_{2}, \widetilde{H}_{3}\right\}=8 I_{2}^{3}, \quad\left\{\widetilde{H}_{3}, H_{4}\right\}=24 I_{2}^{2} H_{4} .
\end{array}
$$

Using this algebra we easily obtain the desired angle variable

$$
\omega_{2}=-\frac{1}{8} \frac{F\left(\frac{I_{2}}{\sqrt{I_{1}}}\right) I_{1}+4 \sqrt{H_{4} I_{2}^{3}+54 I_{1}^{2}}}{I_{2}^{3}}
$$

up to canonical transformation $\omega_{2} \rightarrow \omega_{2}+f\left(I_{2}\right)$. Here $F$ is arbitrary function of the constant of motion, chosen in such a way that relation $\left\{\omega_{1}, \omega_{2}\right\}=0$ is satisfied. 


\section{Case 4}

In case 4 of Theorem 4.1 we have

$$
H_{1}=2 p_{1} p_{2}+q_{1}^{-d-2} q_{2}^{d}
$$

The system generated by this Hamiltonian is integrable for an arbitrary value of parameter $d$ with the following first integral

$$
I_{2}=\left(p_{1} q_{1}-p_{2} q_{2}\right)^{2}-2 \frac{q_{2}^{d+1}}{q_{1}^{d+1}} .
$$

This system admits separation of variables and we show that for rational values of $d$ it is super-integrable.

\subsection{Super-integrability and additive separation of variables}

Let us introduce the following canonical coordinates $(r, \varphi)$ and momenta $\left(p_{r}, p_{\varphi}\right)$ defined by

$$
q_{1}=r(\cosh \varphi+\sinh \varphi)=r e^{\varphi}, \quad q_{2}=r(\cosh \varphi-\sinh \varphi)=r e^{-\varphi},
$$

and

$$
p_{1}=\frac{e^{-\varphi}}{2}\left(p_{r}+\frac{p_{\varphi}}{r}\right), \quad p_{2}=\frac{e^{\varphi}}{2}\left(p_{r}-\frac{p_{\varphi}}{r}\right) .
$$

In new variables Hamiltonian $H_{1}$ and first integral $J_{2}=I_{2} / 2$ take the forms

$$
H_{1}=\frac{p_{r}^{2}}{2}-\frac{p_{\varphi}^{2}}{2 r^{2}}+\frac{e^{-2(d+1) \varphi}}{r^{2}}, \quad J_{2}=\frac{1}{2} p_{\varphi}^{2}-e^{-2(d+1) \varphi} .
$$

New Hamilton's equations read

$$
\begin{array}{ll}
\dot{r}=p_{r}, & \dot{p}_{r}=\frac{-p_{\varphi}^{2}+2 e^{-2(d+1) \varphi}}{r^{3}}, \\
\dot{\varphi}=-\frac{p_{\varphi}}{r^{2}}, & \dot{p}_{\varphi}=\frac{2(d+1) e^{-2(d+1) \varphi}}{r^{2}} .
\end{array}
$$

Let us note that

$$
H_{1}=\frac{1}{2} p_{r}^{2}-\frac{1}{r^{2}} J_{2} \text {. }
$$

In order to perform the explicit integration we introduce as in [4] a new independent variable $\tau$ such that $\mathrm{d} \tau / \mathrm{d} t=1 / r^{2}$. Then we find that

$$
p_{r}=\frac{r^{\prime}}{r^{2}}, \quad p_{\varphi}=-\varphi^{\prime},
$$

where prime denotes the differentiation with respect to $\tau$. In effect we have

$$
H_{1}=\frac{r^{\prime 2}}{2 r^{4}}-\frac{1}{r^{2}} J_{2}, \quad \text { and } \quad J_{2}=\frac{\varphi^{\prime 2}}{2}-e^{-2(d+1) \varphi},
$$


i.e., we effectively separated variables and we can make two independent quadratures

$$
\int \frac{\mathrm{d} r}{\sqrt{2\left(H_{1} r^{4}+J_{2} r^{2}\right)}}=\tau+C_{1}, \quad \int \frac{\mathrm{d} \varphi}{\sqrt{2\left(J_{2}+e^{-2(d+1) \varphi}\right)}}=\tau+C_{2} .
$$

The explicit forms of these elementary integrals are following

$$
\frac{1}{\sqrt{2 J_{2}}} \ln \frac{r}{2\left(\sqrt{J_{2}}+\sqrt{J_{2}+H_{1} r^{2}}\right)}=\tau+C_{1}
$$

and

$$
\frac{1}{(d+1) \sqrt{2 J_{2}}} \operatorname{arcsinh}\left[\sqrt{J_{2}} e^{(d+1) \varphi}\right]=\tau+C_{2} .
$$

Using well-known formula

$$
\operatorname{arcsinh} z=\ln \left[z+\sqrt{z^{2}+1}\right],
$$

one can rewrite the last integral as

$$
\frac{1}{(d+1) \sqrt{2 J_{2}}} \ln \left[e^{(d+1) \varphi} \sqrt{J_{2}}+\sqrt{e^{2(d+1) \varphi} J_{2}+1}\right]=\tau+C_{2} .
$$

From (8.9) and (8.10) we deduce that

$$
\begin{aligned}
& I=(d+1) \sqrt{2 J_{2}}\left(C_{2}-C_{1}\right) \\
& =\ln \left[e^{(d+1) \varphi} \sqrt{J_{2}}+\sqrt{e^{2(d+1) \varphi} J_{2}+1}\right]-(d+1) \ln \frac{r}{2\left(\sqrt{J_{2}}+\sqrt{J_{2}+H_{1} r^{2}}\right)} \\
& =\ln \left[\frac{2^{d+1}}{r^{d+1}}\left(e^{(d+1) \varphi} \sqrt{J_{2}}+\sqrt{e^{2(d+1) \varphi} J_{2}+1}\right)\left(\sqrt{J_{2}}+\sqrt{J_{2}+H_{1} r^{2}}\right)^{d+1}\right],
\end{aligned}
$$

is a first integral of the system. We use relations

$$
J_{2}+H_{1} r^{2}=\frac{1}{2} r^{2} p_{r}^{2}, \quad e^{2(d+1) \varphi} J_{2}+1=\frac{1}{2} e^{2(d+1) \varphi} p_{\varphi}^{2},
$$

to introduce explicitly the momenta and then $I$ takes the form

$$
I=\ln \left[\frac{2^{d / 2}}{r^{d+1}} e^{(d+1) \varphi}\left(\sqrt{2 J_{2}}-p_{\varphi}\right)\left(\sqrt{2 J_{2}}+r p_{r}\right)^{d+1}\right],
$$

We can take as the first integral its exponent, more precisely

$$
H_{3}=2^{-d / 2} \exp (I)=\frac{1}{r^{d+1}} e^{(d+1) \varphi}\left(\sqrt{I_{2}}-p_{\varphi}\right)\left(\sqrt{I_{2}}+r p_{r}\right)^{d+1} .
$$

When we come back to original variables, then this first integral takes the form

$$
H_{3}=\frac{1}{q_{2}^{d+1}}\left(p_{2} q_{2}-p_{1} q_{1}+\sqrt{I_{2}}\right)\left(p_{1} q_{1}+p_{2} q_{2}+\sqrt{I_{2}}\right)^{d+1},
$$


It is easy to notice that

$$
H_{4}=\frac{1}{q_{2}^{d+1}}\left(p_{2} q_{2}-p_{1} q_{1}-\sqrt{I_{2}}\right)\left(p_{1} q_{1}+p_{2} q_{2}-\sqrt{I_{2}}\right)^{d+1} .
$$

is also a first integral. For rational $d$ these functions are algebraic. Thus we can recapitulate these considerations by the following theorem.

Theorem 8.1. Hamiltonian system given by (8.1) is super-integrable with algebraic additional first integral iff $d$ is a rational number.

Using $\mathrm{H}_{3}$ and $\mathrm{H}_{4}$ we can get first integrals which are polynomial with respect to the momenta. For example, if $d$ is positive integer, then we take the following first integrals

$$
\widetilde{H}_{3}=\frac{1}{\sqrt{I_{2}}}\left(H_{3}-H_{4}\right), \quad \widetilde{H}_{4}=H_{3}+H_{4} .
$$

In the sum $H_{3}+H_{4}$ terms containing odd powers of $\sqrt{I_{2}}$ disappear, and, as a result, $\widetilde{H}_{4}$ is a polynomial with respect to the momenta of degree $d+2$. The difference $H_{3}-H_{4}$ contains only odd powers of $\sqrt{I_{2}}$, thus it is divisible by $\sqrt{I_{2}}$, and $\widetilde{H}_{3}$ is polynomial in the momenta of degree $d+1$.

If $d$ is a positive rational number of the form $d=d_{1} / d_{2}$, then we put

$$
\begin{aligned}
& F_{3}=H_{3}^{d_{2}}=\frac{1}{q_{2}^{d_{1}+d_{2}}}\left(p_{2} q_{2}-p_{1} q_{1}+\sqrt{I_{2}}\right)^{d_{2}}\left(p_{1} q_{1}+p_{2} q_{2}+\sqrt{I_{2}}\right)^{d_{1}+d_{2}}, \\
& F_{4}=H_{4}^{d_{2}}=\frac{1}{q_{2}^{d_{1}+d_{2}}}\left(p_{2} q_{2}-p_{1} q_{1}-\sqrt{I_{2}}\right)^{d_{2}}\left(p_{1} q_{1}+p_{2} q_{2}-\sqrt{I_{2}}\right)^{d_{1}+d_{2}},
\end{aligned}
$$

and then

$$
\widetilde{H}_{3}=\frac{1}{\sqrt{I_{2}}}\left(F_{3}-F_{4}\right), \quad \widetilde{H}_{4}=F_{3}+F_{4},
$$

are polynomial first integrals of degrees $d_{1}+2 d_{2}-1$ and $d_{1}+2 d_{2}$, respectively.

In the case when $d \geq-1$ is rational negative, i.e. $d=-d_{1} / d_{2}$, and $d_{2} \geq d_{1}$ where $d_{1}, d_{2} \in \mathbb{N}$ we take

$$
\widetilde{H}_{3}=\frac{1}{\sqrt{I_{2}}}\left(H_{3}^{d_{2}}-H_{4}^{d_{2}}\right), \quad \widetilde{H}_{4}=H_{3}^{d_{2}}+H_{4}^{d_{2}},
$$

as polynomial in the momenta first integrals of degrees $2 d_{2}-d_{1}-1$ and $2 d_{2}-d_{1}$, respectively.

It is not clear if there exist a polynomial in momenta first integral functionally independent with $H_{1}$ and $I_{2}$ for rational $d$ smaller than -1 .

Let us note that potentials from case 4 written in Cartesian coordinates as

$$
V=\frac{(x+\mathrm{i} y)^{k-1}}{(x-\mathrm{i} y)^{k+1}}
$$

were considered in the recent paper [8]. In this paper authors proved the super-integrability of such potentials for $k$ rational for classical and quantum systems. But the explicit form of the additional first integral was not announced. 


\subsection{Examples}

We showed that for rational $d$ the system is super-integrable. We have the second additional first integral $\widetilde{H}_{3}$ which can be chosen as a polynomial with respect to the momenta. Moreover, there exists another additional polynomial integral of motion $\widetilde{H}_{4}$ which can be normalised in such a way that

$$
\widetilde{H}_{4}=\left\{I_{2}, \widetilde{H}_{3}\right\},
$$

satisfying the equations (3.18) as well. For $\kappa_{2}=1$ adding polynomial integrals $\widetilde{H}_{3,4}$ to the generators $\rho, I_{1}, I_{2}$ we obtain the complete algebra of integrals of motion and function $\rho$ defined by the following brackets

$$
\begin{array}{lll}
\left\{I_{1}, \rho\right\}=-2 \alpha I_{1}, & \left\{I_{1}, I_{2}\right\}=0, \quad\left\{I_{1}, \widetilde{H}_{k}\right\}=0, & \left\{\rho, I_{2}\right\}=0, \\
\left\{I_{2}, \widetilde{H}_{3}\right\}=\widetilde{H}_{4}, \quad\left\{\widetilde{H}_{3,4}, \rho\right\}=\widetilde{H}_{3,4}, & \left\{I_{2}, \widetilde{H}_{4}\right\}=2 \kappa^{2} I_{2} \widetilde{H}_{3}, & \left\{\widetilde{H}_{4}, \widetilde{H}_{3}\right\}=\kappa^{2} \widetilde{H}_{3}^{2} .
\end{array}
$$

Here parameter $\kappa$ depends on $\alpha$ and $d$. Let us present some particular examples with cubic polynomial integrals

- for $\alpha=-\frac{1}{3}, \kappa=18, V=\frac{q_{2}^{2}}{q_{1}^{4}}:$

$$
\begin{aligned}
& \widetilde{H}_{3}=2 p_{2}^{3}-2 q_{2} p_{2} q_{1}^{-3}-p_{1} q_{1}^{-2}, \\
& \widetilde{H}_{4}=12 p_{2}^{3}\left(p_{2} q_{2}-p_{1} q_{1}\right)-6 q_{1}^{-3}\left(p_{1}^{2} q_{1}^{2}+p_{1} p_{2} q_{1} q_{2}+4 p_{2}^{2} q_{2}^{2}\right)+\frac{6 q_{2}^{3}}{q_{1}^{6}}, \\
& 36 H_{1}^{3}-\widetilde{H}_{4}^{2}+36 \widetilde{H}_{3}^{2} I_{2}=0,
\end{aligned}
$$

- for $\alpha=-1, \kappa=2, V=\frac{1}{q_{2}^{2 / 3} q_{1}^{4 / 3}}$

$$
\begin{aligned}
& \widetilde{H}_{3}=2 p_{2}\left(p_{2} q_{2}-p_{1} q_{1}\right)^{2}-\frac{2 p_{2} q_{2}-p_{1} q_{1}}{q_{2}^{2 / 3} q_{1}^{1 / 3}} \\
& \widetilde{H}_{4}=4 p_{2}\left(p_{2} q_{2}-p_{1} q_{1}\right)^{3}-\frac{2\left(p_{1} q_{1}-4 p_{2} q_{2}\right)\left(p_{1} q_{1}-p_{2} q_{2}\right)}{q_{1}^{1 / 3} q_{2}^{2 / 3}}+\frac{2}{q_{1}^{2 / 3} q_{2}^{1 / 3}}
\end{aligned}
$$

fourth order integrals

- for $\alpha=-\frac{1}{4}, \kappa=32, V=\frac{q_{2}^{3}}{q_{1}^{5}}$

$$
\begin{aligned}
& \widetilde{H}_{3}=p_{2}^{4}-\frac{3 q_{2}^{2} p_{2}^{2}}{2 q_{1}^{4}}-\frac{q_{2} p_{2} p_{1}}{q_{1}^{3}}-\frac{p_{1}^{2}}{2 q_{1}^{2}}+\frac{q_{2}^{4}}{4 q_{1}^{8}}, \\
& \widetilde{H}_{4}=8 p_{2}^{4}\left(p_{2} q_{2}-p_{1} q_{1}\right)-\frac{4\left(p_{1}^{3} q_{1}^{3}+p_{1}^{2} p_{2} q_{1}^{2} q_{2}+p_{1} p_{2}^{2} q_{1} q_{2}^{2}+5 p_{2}^{3} q_{2}^{3}\right)}{q_{1}^{4}}+\frac{2 q_{2}^{4}\left(3 p_{1} q_{1}+5 p_{2} q_{2}\right)}{q_{1}^{8}}, \\
& 8 H_{1}^{4}-\widetilde{H}_{4}^{2}+64 \widetilde{H}_{3}^{2} I_{2}=0,
\end{aligned}
$$


- for $\alpha=-1, \kappa=2, V=\frac{1}{q_{2}^{3 / 4} q_{1}^{5 / 4}}$

$$
\begin{aligned}
& \widetilde{H}_{3}=p_{2}\left(p_{2} q_{2}-p_{1} q_{1}\right)^{3}+\frac{1}{4 \sqrt{q_{1} q_{2}}}-\frac{\left(3 p_{2} q_{2}-p_{1} q_{1}\right)\left(p_{2} q_{2}-p_{1} q_{1}\right)}{2 q_{2}^{3 / 4} q_{1}^{1 / 4}} \\
& \widetilde{H}_{4}=-2 p_{2}\left(p_{1} q_{1}-p_{2} q_{2}\right)^{4}-\frac{\left(p_{1} q_{1}-5 p_{2} q_{2}\right)\left(p_{1} q_{1}-p_{2} q_{2}\right)^{2}}{q_{1}^{1 / 4} q_{2}^{3 / 4}}+\frac{3 p_{1} q_{1}-5 p_{2} q_{2}}{2 \sqrt{q_{1}} \sqrt{q_{2}}},
\end{aligned}
$$

and fifth order additional integral of motion

- for $\alpha=-\frac{1}{5}, \kappa=50, V=\frac{q_{2}^{4}}{q_{1}^{6}}$

$$
\begin{aligned}
& \widetilde{H}_{3}=\frac{1}{2} p_{2}\left(2 p_{2}^{2}-\frac{q_{2}^{3}}{q_{1}^{5}}\right)\left(2 p_{2}^{2}-\frac{3 q_{2}^{3}}{q_{1}^{5}}\right)-\frac{p_{1}^{3}}{q_{1}^{2}}-\frac{2 q_{2} p_{2} p_{1}^{2}}{q_{1}^{3}}+\frac{q_{2}^{2} p_{1}\left(q_{2}^{3}-3 p_{2}^{2} q_{1}^{5}\right)}{q_{1}^{9}}, \\
& \widetilde{H}_{4}=20 p_{2}^{5}\left(p_{2} q_{2}-p_{1} q_{1}\right)-\frac{10\left(p_{1}^{4} q_{1}^{4}+p_{1}^{3} p_{2} q_{1}^{3} q_{2}+p_{1}^{2} p_{2}^{2} q_{1}^{2} q_{2}^{2}+p_{1} p_{2}^{3} q_{1} q_{2}^{3}+6 p_{2}^{4} q_{2}^{4}\right)}{q_{1}^{5}} \\
& +\frac{5 q_{2}^{5}\left(4 p_{1}^{2} q_{1}^{2}+7 p_{1} p_{2} q_{1} q_{2}+9 p_{2}^{2} q_{2}^{2}\right)}{q_{1}^{10}}-\frac{5 q_{2}^{10}}{q_{1}^{15}}, \\
& 25 H_{1}^{5}-\widetilde{H}_{4}^{2}+100 \widetilde{H}_{3}^{2} I_{2}=0 .
\end{aligned}
$$

According to [23, 25] $\widetilde{H}_{3}$ has to be function on the both action variables $I_{1,2}$ and the single angle variable $\omega_{2}$. Using the complete algebra (8.13) we can easily get

$$
\widetilde{H}_{3}=\left(e^{\kappa \sqrt{2 I_{2}} \omega_{2}}+c e^{-\kappa \sqrt{2 I_{2}} \omega_{2}}\right) I_{1}^{1 / 2 \alpha} .
$$

Here $\omega_{2}$ is defined up to canonical transformation $\omega_{2} \rightarrow \omega_{2}+f\left(I_{2}\right)$ and parameter $c$ is calculated from the bracket $\left\{I_{2}, \omega_{2}\right\}=1$. For instance, for $\alpha=-1 / 3$ and $d=-4$ we have $c=-1 / 16$.

On the other hand, if $\widetilde{H}_{3}$ is the known polynomial solution of (3.18), which satisfies the algebra (8.13), then we can directly calculate $\omega_{2}$

$$
\omega_{2}=\frac{1}{\kappa \sqrt{2 I_{2}}} \ln \left(\frac{\widetilde{H}_{3} I_{2}+\sqrt{\widetilde{H}_{3}^{2} I_{2}^{2}-4 c I_{2} I_{1}^{1 / \alpha}}}{I_{2} I_{1}^{1 / 2 \alpha}}\right)
$$

without separation of variables.

\section{Bi-Hamiltonian systems with higher order integrals of motion}

Let us recall that in Section 4 we investigated systems given by multi-parameter family of Hamiltonian functions given by

$$
H=2 p_{1} p_{2}+q_{1}^{-\frac{\beta(d+1)+\alpha}{\alpha}} q_{2}^{d}
$$


see (4.2). For these systems with arbitrary values of parameters $\alpha, \beta$ and $d$, the corresponding bivector $P^{\prime}$ given by (3.12) is the Poisson bivector compatible, by Proposition 3.1, with canonical bivector $P$. In previous sections we distinguished infinitely many integrable cases with additional first integrals which are polynomial in the momenta of the second degree. Among them infinitely many systems are super-integrable.

In this section we continue our integrability analysis and we look for cases when the additional first integral is a polynomial in momenta of degree greater than two. To this end we solve equations (3.18) with respect to $\mathrm{H}_{2}$ of the form (3.19). The existence of solutions of the obtained equations depends on values of parameters $\alpha, \beta, d$ and $\kappa_{2}$.

Assuming that the additional first integral is of degree 4 in the momenta and setting $\kappa_{2}=1$ we found the following solutions

$$
\begin{aligned}
& \rho=-\frac{1}{4}\left(q_{1} p_{1}+5 p_{2} q_{2}\right), \quad V=q_{1}^{3} q_{2}^{-9 / 5}, \\
& H_{2}=4 p_{1}^{4}-10\left(3 p_{1}^{2} q_{1}^{2}-30 p_{1} p_{2} q_{1} q_{2}+25 p_{2}^{2} q_{2}^{2}\right) q_{2}^{-4 / 5}+225 q_{1}^{4} q_{2}^{-8 / 5}, \\
& \rho=-\frac{1}{6}\left(2 p_{1} q_{1}-p_{2} q_{2}\right), \quad V=q_{1}^{2} q_{2}^{5}, \\
& H_{2}=16 p_{1}^{3}\left(p_{1} q_{1}-p_{2} q_{2}\right)+4 p_{1} q_{1} q_{2}^{6}\left(p_{1} q_{1}-2 p_{2} q_{2}\right)+q_{2}^{8}\left(p_{2}^{2}-q_{1}^{3} q_{2}^{4}\right), \\
& \rho=-\frac{1}{3}\left(p_{1} q_{1}+4 p_{2} q_{2}\right), \quad V=q_{1}^{2} q_{2}^{-7 / 4}, \\
& H_{2}=2 p_{1}^{3}\left(p_{1} q_{1}-p_{2} q_{2}\right)-q_{2}^{-3 / 4}\left(13 p_{1}^{2} q_{1}^{2}-80 p_{1} p_{2} q_{1} q_{2}+64 p_{2}^{2} q_{2}^{2}\right)+64 q_{1}^{3} q_{2}^{-3 / 2}, \\
& \rho=-p_{1} q_{1}-\frac{1}{5} p_{2} q_{2}, \quad V=q_{1}^{-3 / 4} q_{2}^{9 / 4}, \\
& H_{2}=4 p_{1}\left(p_{1} q_{1}-p_{2} q_{2}\right)^{3}-2 q_{1}^{-3 / 4} q_{2}^{-5 / 4}\left(3 p_{1}^{2} q_{1}^{2}-12 p_{1} p_{2} q_{1} q_{2}+p_{2}^{2} q_{2}^{2}\right)+9 q_{1}^{-1 / 2} q_{2}^{-5 / 2} .
\end{aligned}
$$

Assuming that $\mathrm{H}_{2}$ is of sixth degree with respect to the momenta we obtained other variety 
of solutions:

$$
\begin{aligned}
\rho= & -\frac{1}{14}\left(7 p_{1} q_{1}+p_{2} q_{2}\right), \quad V=q_{1}^{-2 / 3} q_{2}^{-10 / 3}, \\
H_{2}= & 4 p_{1}^{2}\left(p_{1} q_{1}-p_{2} q_{2}\right)^{4}+\left(7 p_{1}^{2} q_{1}^{2}-28 p_{1} p_{2} q_{1} q_{2}+p_{2}^{2} q_{2}^{2}\right) q_{1}^{-4 / 3} q_{2}^{-14 / 3} \\
& +2 p_{1}\left(-4 p_{1}^{3} q_{1}^{3}+13 p_{1}^{2} p_{2} q_{1}^{2} q_{2}-20 p_{1} p_{2}^{2} q_{1} q_{2}^{2}+2 p_{2}^{3} q_{2}^{3}\right) q_{1}^{-2 / 3} q_{2}^{-7 / 3}-6 q_{1}^{-1} q_{2}^{-7}, \\
\rho= & -\frac{1}{6}\left(p_{1} q_{1}+7 p_{2} q_{2}\right), \quad V=q_{1}^{2} q_{2}^{-10 / 7}, \\
H_{2}= & 4 p_{1}^{6}-2058 q_{1}^{3} q_{2}^{9 / 7}+343 q_{2}^{-6 / 7}\left(p_{1} q_{1}-7 p_{2} q_{2}\right)\left(p_{1} q_{1}-p_{2} q_{2}\right)+14 p_{1}^{3}\left(-4 p_{1} q_{1}+7 p_{2} q_{2}\right) q_{2}^{-3 / 7}, \\
\rho= & -p_{1} q_{1}+2 p_{2} q_{2}, \quad V=q_{1}^{-2 / 3} q_{2}^{-5 / 6}, \\
H_{2}= & 2 q_{1}^{-2 / 3}\left(p_{1} q_{1}-p_{2} q_{2}\right)^{2}\left(q_{2}^{1 / 6}-2 p_{1} q_{1}^{2 / 3}\left(p_{1} q_{1}-p_{2} q_{2}\right)\right)+q_{1}^{-1 / 3} q_{2}^{-1 / 3}, \\
\rho= & -\frac{1}{5}\left(p_{1} q_{1}+p_{2} q_{2}\right), \quad V=q_{1}^{3 / 2} q_{2}^{-7 / 2}, \\
H_{2}= & q_{1} p_{1}^{6}-p_{2} q_{2} p_{1}^{5}-\frac{5 q_{1}^{3 / 2} p_{1}^{4}}{2 q_{2}^{5 / 2}}+\frac{3 q_{1}^{2} p_{1}^{2}}{2 q_{2}^{5}}+\frac{3 q_{1} p_{1} p_{2}}{4 q_{2}^{4}}+\frac{p_{2}^{2}}{4 q_{2}^{3}}-\frac{q_{1}^{5 / 2}}{8 q_{2}^{15 / 2}} .
\end{aligned}
$$

Here we present one super-integrable system for which equation (3.18) has two functionally independent solutions $\mathrm{H}_{2}$ and $\mathrm{H}_{3}$ :

$$
\rho=-p_{1} q_{1}-\frac{1}{4} p_{2} q_{2}, \quad V=q_{1}^{-2 / 3} q_{2}^{-7 / 3} .
$$

In this case, for $\kappa_{2}=1$, there is fourth order polynomial integral of motion

$$
H_{2}=\frac{p_{1}\left(p_{1} q_{1}-p_{2} q_{2}\right)^{3}}{2}-\frac{13 p_{1}^{2} q_{1}^{2}-44 p_{1} p_{2} q_{1} q_{2}+4 p_{2}^{2} q_{2}^{2}}{16 q_{1}^{2 / 3} q_{2}^{4 / 3}}+q_{1}^{-1 / 3} q_{2}^{-8 / 3}
$$

and for $\kappa_{2}=2$ sixth order polynomial first integral

$$
\begin{aligned}
H_{3} & =4 p_{1}^{2}\left(p_{1} q_{1}-p_{2} q_{2}\right)^{4}+q_{1}^{-4 / 3} q_{2}^{-8 / 3}\left(10 p_{1}^{2} q_{1}^{2}-16 p_{1} p_{2} q_{1} q_{2}+p_{2}^{2} q_{2}^{2}\right) \\
& -4 q_{1}^{-2 / 3} q_{2}^{-4 / 3} p_{1}\left(p_{1} q_{1}-p_{2} q_{2}\right)\left(2 p_{1}^{2} q_{1}^{2}-6 p_{1} p_{2} q_{1} q_{2}+p_{2}^{2} q_{2}^{2}\right)-\frac{3}{q_{1} q_{2}^{4}}
\end{aligned}
$$

In this case the complete algebra of integrals and $\rho$ is given by

$$
\begin{aligned}
& \left\{\rho, H_{1}\right\}=(\alpha+\beta) H_{1}, \quad\left\{\rho, H_{2}\right\}=-H_{2}, \quad\left\{\rho, H_{3}\right\}=-2 H_{3}, \quad\left\{\rho, H_{4}\right\}=-3 H_{4}, \\
& \left\{H_{2}, H_{3}\right\}=H_{4}, \quad\left\{H_{2}, H_{4}\right\}=\frac{3}{8} H_{3}^{2}, \quad\left\{H_{3}, H_{4}\right\}=\frac{27}{8} H_{1}^{4} .
\end{aligned}
$$

REMARK 9.1 According to our knowledge it is the first example of super-integrable system with first integrals of degree two, four and six with respect to the momenta. In all examples known to us super-integrable systems have at least two additional first integrals of degree two with respect to the momenta [5, 14, 21, 24, 25]. 


\subsection{Additive separation of variables without super-integrability}

Let us consider one of the bi-Hamiltonian systems listed above

$$
\begin{aligned}
& H_{1}=2 p_{1} p_{2}+q_{1}^{3} q_{2}^{-9 / 5}, \quad \rho=-\frac{1}{4}\left(q_{1} p_{1}+5 p_{2} q_{2}\right), \quad \kappa_{2}=1, \\
& H_{2}=4 p_{1}^{4}-10\left(3 p_{1}^{2} q_{1}^{2}-30 p_{1} p_{2} q_{1} q_{2}+25 p_{2}^{2} q_{2}^{2}\right) q_{2}^{-4 / 5}+225 q_{1}^{4} q_{2}^{-8 / 5} .
\end{aligned}
$$

In this case second Poisson bivector is equal to

$$
P^{\prime}=\mathcal{L}_{\rho X} P=\frac{1}{2}\left(\begin{array}{cccc}
0 & q_{1} p_{1}-5 q_{2} p_{2} & p_{1} p_{2}-\frac{3 q_{1}^{3}}{2 q_{2}^{9 / 5}} & 5 p_{2}^{2}+\frac{9 q_{1}^{4}}{10 q_{2}^{14 / 5}} \\
* & 0 & p_{1}^{2}-\frac{15 q_{1}^{2}}{2 q_{2}^{4 / 5}} & 5 p_{1} p_{2}+\frac{9 q_{1}^{3}}{2 q_{2}^{9 / 5}} \\
* & * & 0 & -\frac{3 q_{1}^{2}}{10 q_{2}^{14 / 5}}\left(3 q_{1} p_{1}+25 q_{2} p_{2}\right) \\
* & * & * & 0
\end{array}\right)
$$

and the corresponding recursion operator $\mathcal{N}=P^{\prime} P^{-1}$ is degenerated

$$
\operatorname{det}(\mathcal{N}-\lambda \mathrm{Id})=\lambda^{2}\left(\lambda-\frac{3}{2} H_{1}\right)^{2}
$$

According to [26], we have to look for another solution $P^{\prime}$ of the equations (3.1) associated with the non-trivial Darboux-Nijenhuis variables. The existence of such solution converts $\mathbb{R}^{4}$ into a regular bi-Hamiltonian manifold.

It is easily to find three different second order polynomial solutions of equations (3.1). Only one of them allows us to get variables of separation. Namely, if

$$
Z^{1}=-4 q_{2} p_{1}^{2}, \quad Z^{2}=0, \quad Z^{3}=25 q_{2}^{1 / 5}\left(3 p_{1} q_{1}+5 p_{2} q_{2}\right),
$$

and

$$
Z^{4}=-5 q_{2}^{-6 / 5}\left(\left(2 p_{1} p_{2} q_{2}^{9 / 5}+q_{1}^{3}\right) \sqrt{10}-3 p_{1} q_{2}^{2 / 5} q_{1}^{2}-15 p_{2} q_{2}^{7 / 5} q_{1}\right)
$$

are coordinates of the vector field $Z=\sum Z^{k} \partial_{k}$, then bivector

$$
P^{\prime}=\mathcal{L}_{Z} P
$$

is the solution of (3.1) for the given integrable system. For this Poisson bivector $P^{\prime}$ the control matrix $F$

$$
P^{\prime} \mathrm{d} H_{i}=P \sum_{j=1}^{2} F_{i j} \mathrm{~d} H_{j}, \quad i=1,2,
$$

is a non-degenerate matrix of the form

$$
F=\left(\begin{array}{cc}
10 q_{2}^{1 / 5}\left(-15 q_{1}+q_{2}^{2 / 5} \sqrt{10} p_{1}\right) & \frac{1}{2} \\
-500 q_{2}^{1 / 5}\left(2 q_{2} p_{1}^{2}+15 q_{2}^{1 / 5} q_{1}^{2}-3 q_{2}^{3 / 5} \sqrt{10} p_{1} q_{1}+5 q_{2}^{8 / 5} \sqrt{10} p_{2}\right) & 0
\end{array}\right)
$$


The eigenvalues $u_{1,2}$ of $F$

$$
\begin{aligned}
& A(\lambda)=\left(\lambda-u_{1}\right)\left(\lambda-u_{2}\right)= \\
& =\lambda^{2}-10 q_{2}^{1 / 5}\left(q_{2}^{2 / 5} \sqrt{10} p_{1}-15 q_{1}\right) \lambda+250 q_{2}^{2 / 5}\left(15 q_{1}^{2}+\sqrt{10} q_{2}^{2 / 5}\left(5 p_{2} q_{2}-3 q_{1} p_{1}\right)+2 p_{1}^{2} q_{2}^{4 / 5}\right),
\end{aligned}
$$

coincide with eigenvalues of the corresponding recursion operator $\mathcal{N}=P^{\prime} \cdot P^{-1}=\mathcal{L}_{Z} P$. $P^{-1}$. Thus $u_{1,2}$ are the variables of separation and the so-called Darboux-Nijenhuis coordinates. The conjugated momenta $v_{1,2}$ are equal to

$$
v_{1,2}=-\frac{q_{1}}{40 \sqrt{10} q_{2}^{3 / 5}}-\frac{p_{1}}{200 q_{2}^{1 / 5}} \pm \frac{u_{1}-u_{2}}{200 q_{2}^{4 / 5}}, \quad\left\{u_{j}, v_{k}\right\}=\delta_{i j} .
$$

The separated equations look like

$$
-2\left(\sigma v_{k}\right)^{3} u_{k}+H_{2}+2 u_{k} H_{1}=0, \quad k=1,2, \quad \sigma=10^{3 / 2} .
$$

Thus, in order to get solutions $v_{k}\left(t, \beta_{1}, \beta_{2}\right)$ and $u_{k}\left(t, \beta_{1}, \beta_{2}\right)$ we have to solve the following Abel-Jacobi equations

$$
\begin{aligned}
\beta_{1}-t & =H_{2} \int^{v_{1}} \frac{\mathrm{d} v}{\left(\sigma^{3} v^{3}+H_{1}\right)^{2}}+H_{2} \int^{v_{2}} \frac{\mathrm{d} v}{\left(\sigma^{3} v^{3}+H_{1}\right)^{2}} \\
\beta_{2} & =\int^{v_{1}} \frac{\mathrm{d} v}{2\left(\sigma^{3} v^{3}-H_{1}\right)}+\int^{v_{2}} \frac{\mathrm{d} v}{2\left(\sigma^{3} v^{3}-H_{1}\right)} .
\end{aligned}
$$

There are no addition theorem for the above quadratures and, therefore, we can suppose, that there are no additional polynomial integrals of motion [24, 25]. In fact, the second angle variable

$$
\begin{aligned}
\omega_{2} & =\frac{1}{2} \sum_{k=1}^{2} \int^{v_{k}} \frac{\mathrm{d} v}{\sigma^{3} v^{3}-H_{1}}=\frac{1}{12 \sigma H_{1}^{2 / 3}} \sum_{k=1}^{2}\left[2 \ln \left(\sigma v_{k}-H_{1}^{1 / 3}\right)\right. \\
& \left.-\ln \left(\sigma^{2} v_{k}^{2}+\sigma v_{k} H_{1}^{1 / 3}+H_{1}^{2 / 3}\right)-2 \sqrt{3} \arctan \left(\frac{2 \sigma v_{k}+H_{1}^{1 / 3}}{\sqrt{3} H_{1}^{1 / 3}}\right)\right]
\end{aligned}
$$

cannot be rewritten as a function on polynomials $H_{1}, H_{2}$ and $H_{3}$ as in the super-integrable case (8.14).

In the similar manner we can get solutions of the equations of motion for other systems with fourth order integral of motion. Construction of the variables of separation for the systems with sixth order integrals of motion is an open problem.

\section{Acknowledgements}

AJM and MP thank very much Artur Sergyeyev and Maciej Błaszak for discussions related to this work. For them this research has been partially supported by grant No. N N202 212633 of Ministry of Science and Higher Education of Poland. For AJM this research has been also partially supported by EU funding for the Marie-Curie Research Training Network AstroNet. 


\section{A Appendix. Hypergeometric equation}

Hypergeometric equation

$$
z(1-z) w^{\prime \prime}+[c-(a+b+1) z] w^{\prime}-a b w=0,
$$

is a special case of the Riemann $P$ equation. Thus it possesses three regular singularities at $z=0, z=1$ and $z=\infty$, with exponents and their differences of the following form

$$
\begin{array}{llll}
z=0, & \rho_{1}=1-c, & \rho_{2}=0, & \lambda=1-c, \\
z=1, & \sigma_{1}=c-a-b, & \sigma_{2}=0, & v=c-a-b, \\
z=\infty, & \tau_{1}=b, & \tau_{2}=a, & \mu=b-a .
\end{array}
$$

Exponents satisfy the Fuchs relation, i.e. their sum is equal to one.

As it is well known one of solutions of equation (A.1) holomorphic at the origin is the hypergeometric function given by the following series

$$
{ }_{2} F_{1}(a, b, c, z):=\sum_{k=0}^{\infty} \frac{(a)_{k}(b)_{k}}{(c)_{k} k !} z^{k} .
$$

Here $(x)_{k}=x(x+1) \cdots(x+k)$ is the Pochhammer symbol and $(x)_{0}=1$. The above series is a polynomial iff $a$ or $b$ is a non-positive integer, see, e.g., [18, 7].

The following lemma gives the necessary and sufficient condition for (A.1) to be reducible. It is a classical, well known fact, see, e.g., [7].

Lemma A.1. Equation (A.1) is reducible if and only there exist $i, j, k \in\{1,2\}$, such that

$$
\rho_{i}+\sigma_{j}+\tau_{k} \in \mathbb{Z}
$$

Equivalently, equation (A.1) is reducible if and only if at least one number among

$$
\lambda+\mu+v, \quad-\lambda+\mu+v, \quad \lambda-\mu+v, \quad \lambda+\mu-v,
$$

is an odd integer.

From the above lemma it follows that if equation (A.1) is reducible, then we can always renumber exponents in such a way that

$$
\rho_{1}+\sigma_{1}+\tau_{1} \in-\mathbb{N}_{0},
$$

where $\mathbb{N}_{0}$ denotes the set of non-negative integers. But then, from the Fuchs relation, we also have

$$
\rho_{2}+\sigma_{2}+\tau_{2} \in \mathbb{N} \text {. }
$$

Hence, if A.1 is reducible, we assume from now on that the exponents are numbered in this way.

If the difference of exponents at a singular point is an integer, then it can happen that a local solution around this singularity contains a logarithm. Such a singularity is called logarithmic. For equation (A.1), it is enough to know the exponents to decide which singularity is logarithmic. To formulate the next lemma which gives the necessary and 
sufficient conditions for a singularity of A.1) to be logarithmic we introduce the following notation. For a non-negative integer $m \in \mathbb{N}_{0}$ we define

$$
\langle m\rangle:= \begin{cases}\varnothing & \text { if } m=0, \\ \{1, \ldots, m\} & \text { otherwise }\end{cases}
$$

For $s \in\{0,1, \infty\}$ let $e_{s, 1}$ and $e_{s, 2}$ denote exponents of equation (A.1), ordered in such a way that $\operatorname{Re} e_{s, 1} \geq \operatorname{Re} e_{s, 2}$. With the above notation we have the following.

Lemma A.2. Let $r \in\{0,1, \infty\}$. Then $r$ is a logarithmic singularity of equation (A.1) if and only if $m:=e_{r, 1}-e_{r, 2} \in \mathbb{N}_{0}$, and

$$
e_{r, 1}+e_{s, i}+e_{t, j} \notin\langle m\rangle, \text { for } i, j \in\{1,2\},
$$

where $r, s, t$ are pairwise different elements of $\{0,1, \infty\}$.

For more details, see Lemma 4.7 and its proof on pp. 91-93 in [7].

For considerations of this paper we need characterisation when reducible hypergeometric equation has algebraic solutions. The answer is contained in the following theorem.

Theorem A.3. Assume that hypergeometric equation (A.1) is reducible. Then all its solutions are algebraic iff

1. all exponents are rational, and

2. exactly two or four of $\lambda+\mu+v,-\lambda+\mu+v, \lambda-\mu+v, \lambda+\mu-v$ are odd integers, and

3. no one of singularities is logarithmic.

For proof and details see [9, 7, 18].

\section{References}

[1] M. Audin. Les systèmes hamiltoniens et leur intégrabilité. Cours Spécialisés 8, Collection SMF. SMF et EDP Sciences, Paris, 2001.

[2] J. Bertrand, Théorème relatif au mouvement d'un point attiré vers un centre fixe. $C$. R. Acad. Sci. Paris v. LXXVII, pp. 849-853, 1873.

[3] O. I. Bogoyavlenskij. Algebraic properties of master symmetries and their applications. Dokl. Akad. Nauk, v. 360(4), pp. 445-447, 1998.

[4] A. V. Borisov, A. A. Kilin, and I. S. Mamaev. Superintegrable Systems on a Sphere with Integral of Higher Degree. Regul. Chaotic Dyn., v.14 (6), pp. 615-620, 2009.

[5] C. Daskaloyannis, K. Ypsilantis, Unified treatment and classification of superintegrable systems with integrals quadratic in momenta on a two-dimensional manifold, J. Math. Phys., v.47, 2006, p. 042904, 38 pages, arXiv:math-ph/0412055v3. 
[6] Yu.A. Grigoryev, A.V. Tsiganov, Symbolic software for separation of variables in the Hamilton-Jacobi equation for the L-systems, Regul. Chaotic Dyn., v. 10(4), pp. 413-422, 2005.

[7] K. Iwasaki, H. Kimura, S. Shimomura, and M. Yoshida, From Gauss to Painlevé, A modern theory of special functions, Aspects of Mathematics, E16. Friedr. Vieweg \& Sohn, Braunschweig, 1991.

[8] E. G. Kalnins, W. Miller Jr., G. S. Pogosyan, Superintegrability and higher order constants for classical and quantum systems, arXiv:0912.2278v1 [math-ph], 24 pages.

[9] T. Kimura, On Riemann's equations which are solvable by quadratures. Funkcial. Ekvac., v. 12, pp. 269-281, 1969/1970.

[10] A. J. Maciejewski, M. Przybylska, All meromorphically integrable 2D Hamiltonian systems with homogeneous potential of degree 3, Phys. Lett. A, v. 327(5-6), pp. 461473, 2004.

[11] A. J.Maciejewski, M. Przybylska, Darboux points and integrability of Hamiltonian systems with homogeneous polynomial potential, J. Math. Phys., v. 46(6), p. 062901, 33 pages, 2005.

[12] A. J. Maciejewski, M. Przybylska, H. Yoshida, Necessary conditions for superintegrability of Hamiltonian systems. Phys. Lett. A, v. 372(34), pp. 5581-5587, 2008.

[13] A. J. Maciejewski, M. Przybylska, Differential Galois theory and Integrability. Internat. J. Geom. Methods in Modern Phys., v. 6(8), pp. 1357-1390, 2009.

[14] I. Marquette, P. Winternitz, Polynomial Poisson algebras for classical superintegrable systems with a third-order integral of motion, J. Math. Phys. v.48, p. 012902, 2007.

[15] J. J. Morales Ruiz. Differential Galois Theory and Non-Integrability of Hamiltonian Systems, volume 179 of Progress in Mathematics. Birkhäuser Verlag, Basel, 1999.

[16] J. J. Morales-Ruiz, J. P. Ramis, A note on the non-integrability of some Hamiltonian systems with a homogeneous potential, Methods Appl. Anal., v. 8(1), pp. 113-120, 2001.

[17] K. Nakagawa, Direct construction of polynomial first integrals for Hamiltonian systems with a two-dimensional homogeneous polynomial potential, Ph.D. thesis, The Graduate University for Advanced Studies, Japan, 2002.

[18] E. G. C. Poole, Introduction to the theory of linear differential equations. Dover Publications Inc., New York, 1960

[19] M. Przybylska, Darboux points and integrability of homogenous Hamiltonian systems with three and more degrees of freedom. Regul. Chaotic Dyn., v. 14(2), pp. 263311, 2009.

[20] M. Przybylska, Darboux points and integrability of homogenous Hamiltonian systems with three and more degrees of freedom. Nongeneric cases. Regul. Chaotic Dyn., v. 14(3), pp. 349-388, 2009. 
[21] M.F. Ranada, M. Santander, Complex Euclidean super-integrable potentials, potentials of Drach, and potential of Holt, Phys. Lett. A, v. 278, pp. 271-279, 2001.

[22] A. V. Tsiganov, Towards a classification of natural integrable systems, Regul. Chaotic Dyn., v. 11(3), pp. 343-362, 2006.

[23] A.V. Tsiganov, On maximally superintegrable systems, Regul. Chaotic Dyn., v. 13, pp. 178-190, 2008.

[24] A.V. Tsiganov, Addition theorem and the Drach superintegrable systems, J. Phys. A: Math. Theor., v. 41(33), p. 335204 (16pp), 2008.

[25] A.V. Tsiganov, Leonard Euler: addition theorems and superintegrable systems, Regul. Chaotic Dyn., v. 14(3), pp. 389-406, 2009.

[26] A.V. Tsiganov, On bi-integrable natural Hamiltonian systems on the Riemannian manifolds, arXiv:1006.3914, accepted to Journal of Nonlinear Mathematical Physics, 2010. 